\title{
Norois
}

Environnement, aménagement, société

$242 \mid 2017$

Espace fluvial, ancrage territorial, travail collaboratif, réseau

\section{L'arganeraie marocaine, un système traditionnel face aux mutations récentes : le cas du territoire des Haha, Haut Atlas occidental}

The Moroccan arganeraie, a traditional system in the face of recent changes: case of Haha (Western High Atlas)

\section{Hassan Faouzi}

\section{OpenEdition}

Journals

\section{Édition électronique}

URL : https://journals.openedition.org/norois/6048

DOI : $10.4000 /$ norois. 6048

ISBN : 978-2-7535-5895-3

ISSN : $1760-8546$

\section{Éditeur}

Presses universitaires de Rennes

Édition imprimée

Date de publication : 22 juin 2017

Pagination : $57-81$

ISBN : 978-2-7535-5893-9

ISSN : 0029-182X

\section{Référence électronique}

Hassan Faouzi, «L'arganeraie marocaine, un système traditionnel face aux mutations récentes : le cas du territoire des Haha, Haut Atlas occidental », Norois [En ligne], 242 | 2017, mis en ligne le 22 juin 2019, consulté le 13 janvier 2022. URL : http://journals.openedition.org/norois/6048 ; DOI : https:// doi.org/10.4000/norois.6048 


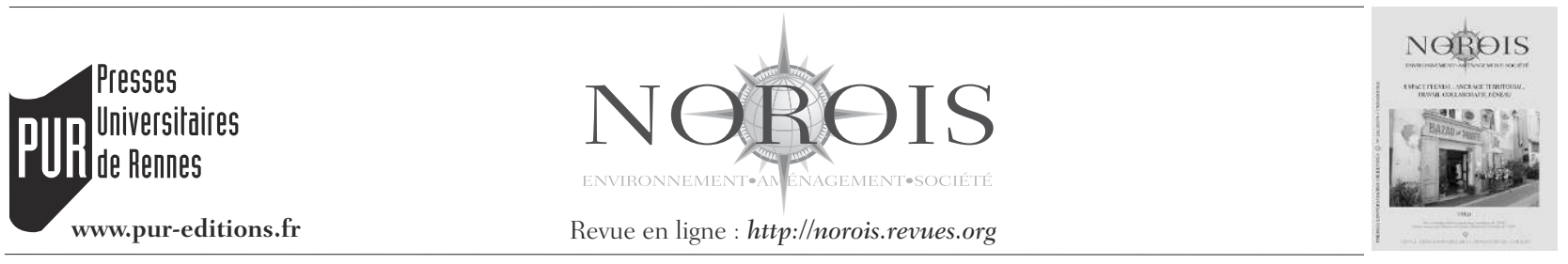

\title{
L'arganeraie marocaine, un système traditionnel face aux mutations récentes : le cas du territoire des Haha, Haut Atlas occidental
}

\author{
The Moroccan Arganeraie, a Traditional System in the Face of Recent Changes: \\ Case of Haha (Western High Atlas)
}

\author{
Hassan FAOUZI
}

Université Internationale d'Agadir, Universiapolis, Maroc - Laboratoire 2L2S, université de Lorraine, Metz, France/. (hassan.faouzi@universiapolis.ma)

\begin{abstract}
Résumé : L'arganeraie est un écosystème d'une grande importance écologique, culturelle et économique. Son modèle socio-économique est resté traditionnel, organisé d'une part autour d'une structure socio-juridique particulière et qui fonctionne d'autre part sur la base de productions variées. L'huile d'argane est la production la plus notoire de l'arganeraie des Haha, mais la croissance démographique, combinée aux conditions naturelles difficiles et à une exploitation supérieure aux capacités de production, rend de plus en plus difficile l'application des règles traditionnelles de gestion durable. Cela implique des mutations socio-spatiales qui déstabilisent un système fragile. L'arganeraie des Haha; célèbre confédération de tribus du Haut-Atlas occidental, en est l'exemple type.

Abstract: The Argan tree is an ecosystem of great ecological, cultural and economic importance. Its socio-economic model has remained traditional, organized on the one hand around a particular socio-legal structure and works on the other hand on the basis of various productions. Argan oil is the most well-known production of the region, but it is not the only one and this set provides the basis for the daily lives of the arganeraie population, the Berbers. These local populations have for so long put in place subtle management practices that have allowed a sustainable use of the argan tree which was sometimes the only recourse for the population survival. But the current population growth, together with the harsh natural conditions and with exploitation in excess of the production capacities, make it more and more difficult the application of the traditional rules of sustainable management; henceforth operating profound changes both in space and in the society making the system equilibrium very fragile. The argan tree of Haha, famous tribe of the Western High Atlas, is a typical example.
\end{abstract}

Mots clés : système agraire - agroforêts - dégradation de l'environnement - Maroc

Keywords: agrarian systems - agroforest - environmental degradation - Morocco

\section{INTRODUCTION}

Le système agraire de l'arganeraie des Haha s'articule autour de l'arganier et sa production de noix d'argane, l'élevage de petits ruminants, surtout des caprins, et la culture de céréales, principalement l'orge. L'occupation de l'espace obéit à des règles complexes issues de la juxtaposition de trois législations : la législation forestière, qui distingue le territoire privé du territoire domanial, la loi coranique, qui définit les règles d’héritage, et le droit coutumier, qui organise, au sein du territoire domanial, la répartition des parcelles entre les usagers et leur utilisation au cours de l'année. 
Autrefois, et jusqu'au début du $\mathrm{Xx}^{\mathrm{e}}$ siècle, cette arganeraie donnait lieu à une exploitation beaucoup plus systématique qu'aujourd'hui. Elle constituait parfois l'unique recours pour la survie de la population. Le pâturage, la céréaliculture en bour, huile d'argane et le bois étaient les principales ressources fournies par l'arganeraie et elles favorisaient le maintien en place de cette population rurale aux conditions très modestes. L'arganier, arbre sacré, a toujours été considéré comme un bien familial et d'ailleurs il a toujours eu une valeur sentimentale. L'exploitation traditionnelle de l'arganeraie était la seule pratique qui existait et qui fournissait même un revenu appréciable en préservant les peuplements d'arganiers. À l'heure actuelle, la mondialisation, la pression démographique et l'expansion de l'agriculture ont tendance à détruire ce patrimoine. En effet, on assiste ces dernières années, au sein de cette forêt, à un changement dans la gestion de l'espace (Faouzi, 2003).

Le présent article a pour objectif d'analyser l'impact de ces mutations récentes, tant sur les populations locales que sur les ressources à partir de l'exemple de l'arganeraie des Haha. Dans cet article, nous nous interrogeons aussi sur la durabilité de l'ar- ganeraie et également sur la question de savoir si les prochaines générations verront-elles encore des forêts d'arganiers et si elles pourront aspirer à un meilleur niveau de vie que leurs parents.

Pour répondre à ces questions, notre analyse s'appuie sur différentes approches scientifiques: recueil de données écologiques, une série de photographies aériennes, photos satellite, observations sur le terrain, enquêtes et entretiens avec la population locale et les acteurs locaux. Le choix de l'arganeraie des Haha s'explique par le fait que c'est dans celle-ci que la problématique de la recherche avait été établie et que des travaux avaient été entrepris antérieuerement (Benzyane, 1989; Ponteves, 1989; Person, 1998; Faouzi, 2003). l'arganeraie des Haha souffre d'un certain nombre de handicaps tant physiques qu'humains qui rendent compte de sa transformation progressive et de l'ouverture tardive vers le monde extérieur de cette région restée longtemps marginalisée, enclavée, à l'écart des principaux axes de circulation. Si l'axe nord-sud qui relie le nord et le sud du Maroc traverse les deux principaux centres ruraux des Haha qui sont Tamanar et Smimou, il ne permet pas de relier les autres régions des Haha aux centres urbains (figures 1 et 2). Dans cette zone du

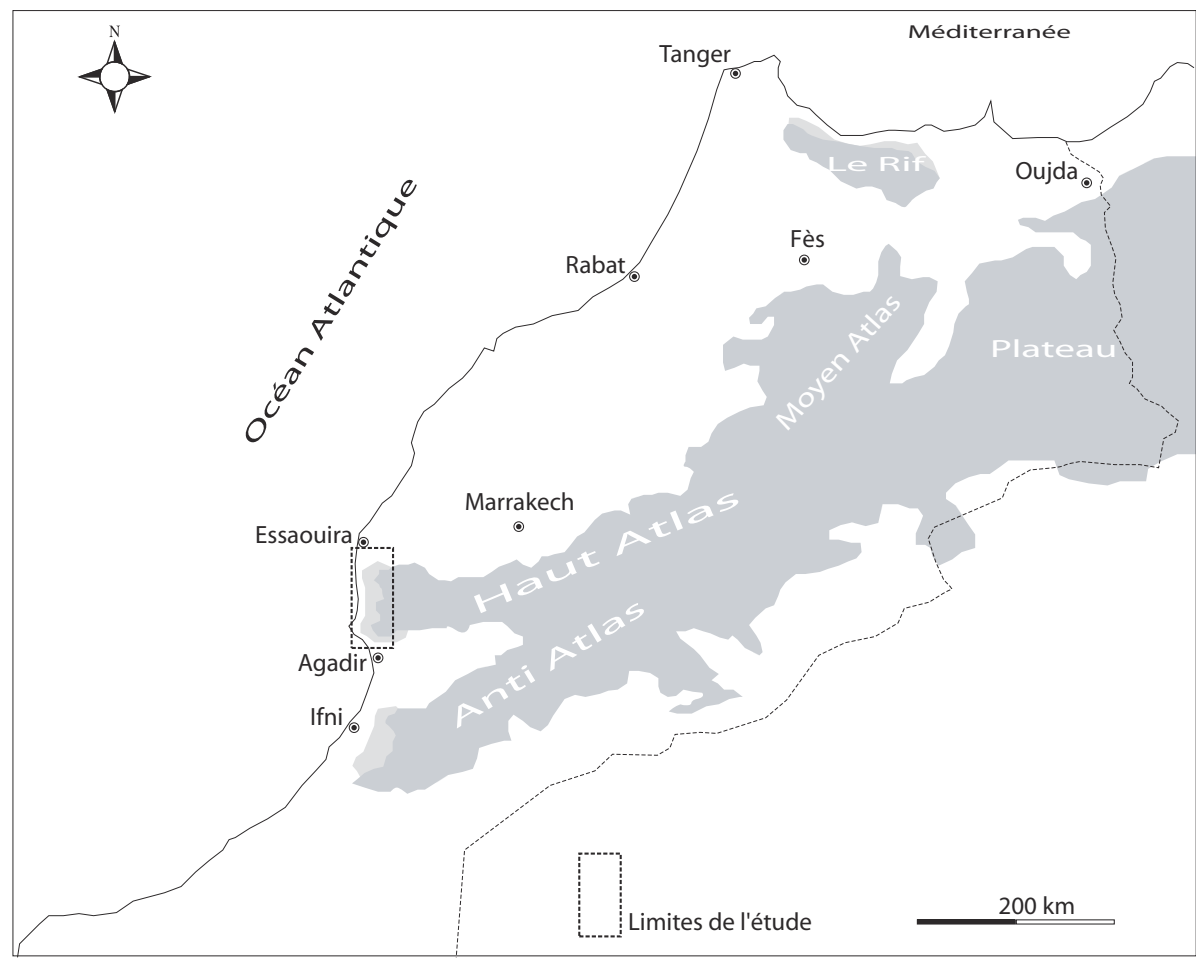

Figure 1 : Localisation de la région d'étude area Location of the study 


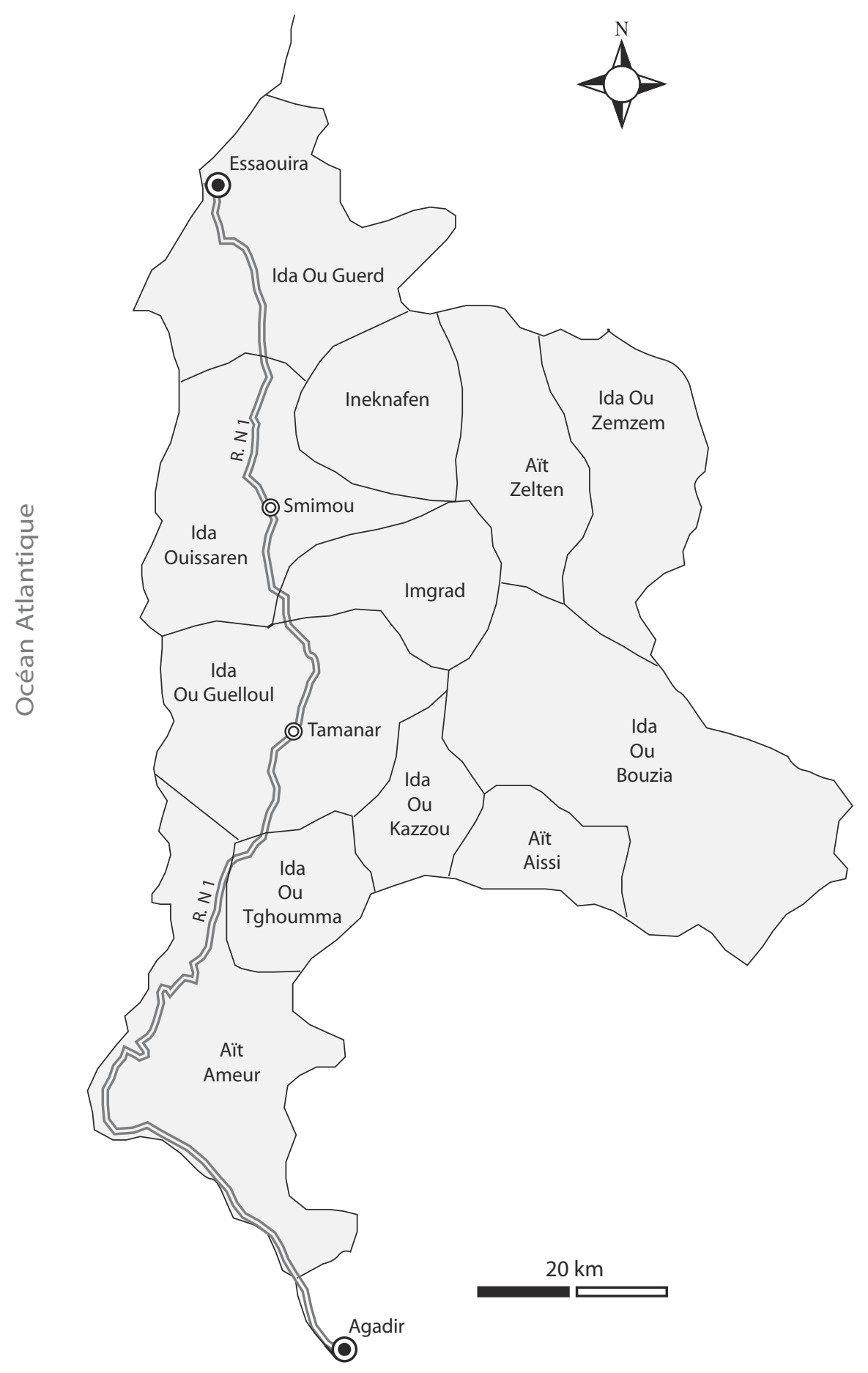

Maroc, région de vieille sédentarisation, où vivent environ 190218 habitants (Haut Commissariat du Plan, 2004), les caractéristiques socio-économiques et les conditions de vie deviennent de plus en plus dures; phénomène qui s'est accentué avec les récentes années de sécheresse.

\section{DES CONDITIONS NATURELLES DIFFICILES}

Le pays des Haha est un pays de basses montagnes, très disséqué et peu favorable à l'installation humaine. Cette région correspond au terroir 
de la célèbre confédération de tribus du Haut-Atlas Occidental : les Haha (figure 2). Sur le plan physique, il s'agit d'un plateau dont l'altitude varie entre 500 et $1600 \mathrm{~m}$.

Selon Emberger (1954), la région est classée du point de vue bioclimatique comme une zone à climat semi-aride. En effet, face à une forte évaporation, la pluviométrie moyenne (250 à 300 mm/an) reste malgré tout, non seulement faible, mais aussi sans grande signification puisque très irrégulière.

Dans cette région l'aridité constitue un facteur défavorable, surtout si l'on en croit les autochtones qui insistent sur une dégradation climatique notable depuis une soixantaine d'années. Ceci est corroboré par les statistiques climatiques officielles. Une analyse de l'évolution de la température ainsi que de la variabilité temporelle de la pluviométrie, pour plusieurs stations (Essaouira, Imgrad, Tamanar, Boutazert), fait ressortir une élévation de la température moyenne de l'ordre de $2{ }^{\circ} \mathrm{C}$ ainsi qu'une baisse très importante d'environ $30 \%$ du cumul des précipitations durant la période 1978-1994 par rapport à la période 1961-1977. La saison 1994-1995 a été la plus sèche du siècle au Maroc (Bahir et al., 2002).

Les sécheresses prolongées des années 1980, 1990 et 2000 ont entraîné un abaissement considé- rable de la nappe phréatique déjà en situation très critique. De nombreux puits traditionnels de type naora ou sanya ont tari. Ceux qui étaient équipés de motopompes ont été abandonnés. La pénurie d'eau est devenue un réel problème.

Le rôle du climat et du milieu physique est parmi les facteurs déterminants de l'organisation de l'espace des Haha. Selon la direction provinciale d'agriculture d'Essaouira (2001), la campagne agricole 2000/2001 marquée par une forte sécheresse, a vu l'espace agricole se rétracter de $47 \%$ par rapport à une année normale. Dans nos discussions avec les fellahs, on a relevé plusieurs signes de difficultés pour faire face aux aléas climatiques.

Dans cette région pauvre, au climat difficile, l'assèchement récent du climat se traduit aussi par une mobilisation des éléments fins par le vent. La situation est aggravée par le vent du nord, quasi permanent et fort : 300 jours par an, d'où le déplacement des dunes et d'où l'effet néfaste de l'ensablement : atteinte aux sites agricoles et aux puits, etc. (figure 3).

Partout chez les Haha on observe des barkhanes très mobiles ainsi que des dunes aux formes variées. Certes, ce phénomène n'est pas nouveau, puisqu'il avait déjà été évoqué par Sulzlee dans son article

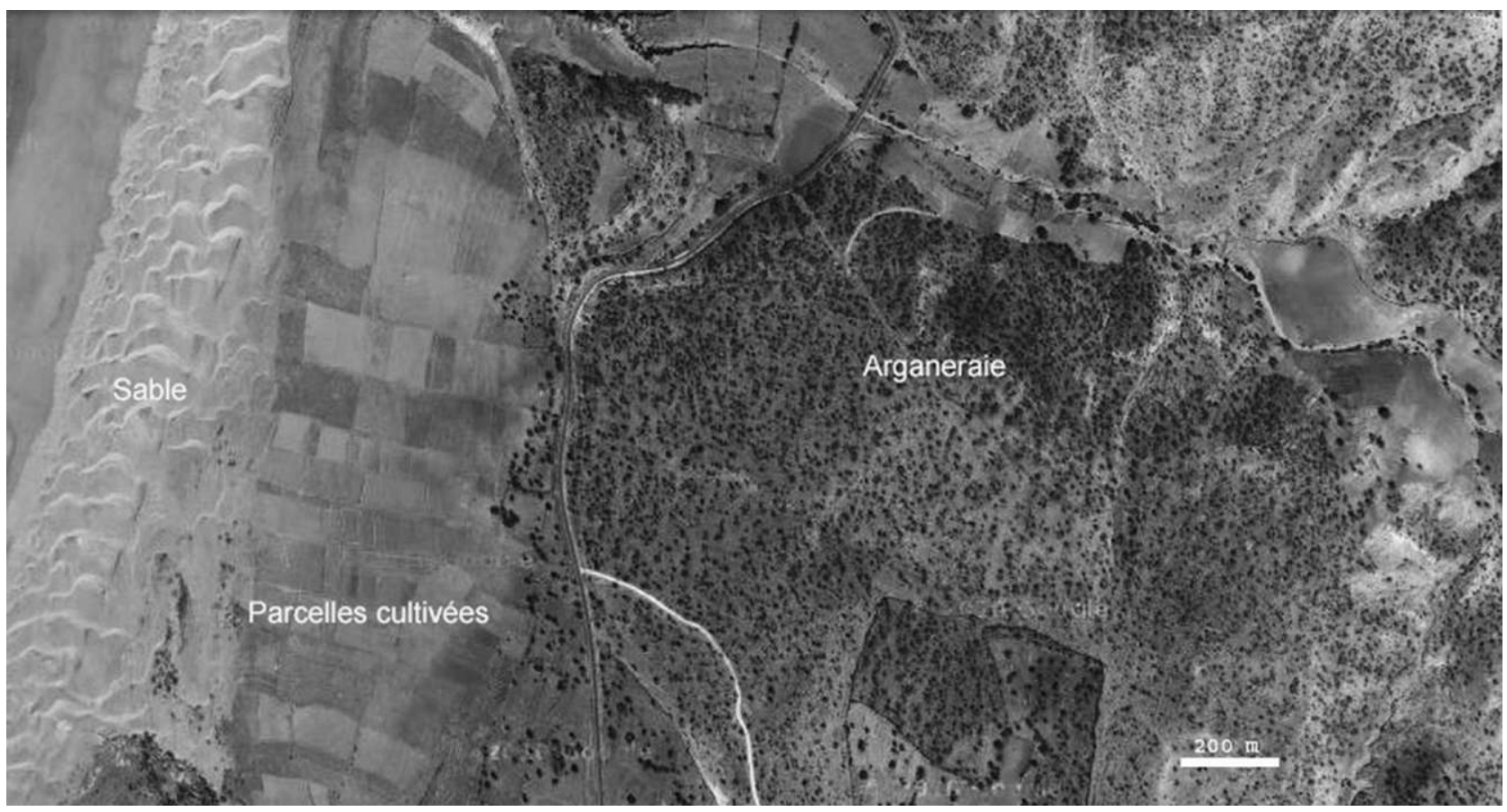

Figure 3 : Photo satellite montrant des parcelles envahies par le sable à Cap Tafelney (source : Google-Imagerie TERRAMetrics, 2011) Satellite photo showing parcels invaded by sand at Tafelney Cap 
intitulé «Les dunes d'Essaouira » paru en 1962 dans la revue forestière française. Mais en plusieurs années d'observations dans le nord-ouest des Haha, on note une augmentation du stock de sable disponible. On y observe de nombreux épandages de sable, d'ordre décamétrique qui sont dangereux pour les arganiers et qui menacent les espaces agricoles déjà limités.

En plus des faibles ressources naturelles et de l'isolement relatif des Haha, les systèmes économiques traditionnels et ancestraux, tournés vers l'autoconsommation, connaissent une crise qui résulte de la combinaison de plusieurs facteurs. Sans faire une analyse détaillée, on peut citer par exemple la faiblesse des productions agricoles sur un espace cultivé de plus en plus restreint, les structures foncières avec des propriétés souvent très morcelées et dont la superficie totale est inférieure dans $80 \%$ des cas à 5 ha $(77,75 \%$ dans la commune d'Imgrad, 86,67\% dans la commune d'Ida Ou Throumma, $91 \%$ dans la commune des Ait Daoud, $83 \%$ dans la commune de Smimou) (Faouzi, 2003).

Le morcellement de l'espace trouve son explication dans l'éclatement des structures familiales (un père de famille a le devoir d'attribuer une partie de ses terres à ses enfants devenant indépendants après le mariage). Parfois aussi, en raison de l'indisponibilité des terres (l'ensemble du terroir étant approprié) la seule possibilité pour les jeunes exploitations d'accéder à un lopin de terre est l'héritage ou l'emprunt de parcelles. Le processus d'occupation de l'espace montre clairement l'intensité de la pression foncière. Par conséquent, les règles traditionnelles de gestion foncière basées sur la gestion collective des terres lignagères sont révolues.

En plus du morcellement de la propriété, la concurrence de l'urbanisation a fait disparaître de nombreuses parcelles encore cultivées au début des années 1990. Une seconde manifestation de cette concurrence, moins visible dans le paysage, est celle qui s'exerce pour la ressource en eau, entraînant ainsi de profondes transformations de l'espace hihi.

\section{UN SYSTÈME TRADITIONNEL EN PLEINE MUTATION}

\section{Démographie et émigration : deux facteurs d'évolution majeurs}

L'importance numérique de la population et sa répartition dans l'espace ont un grand intérêt géographique dans la mesure où la pression démographique figure parmi les facteurs ayant occasionné un déséquilibre socio-économique de la région des Haha. Cet espace ne pouvant plus nourrir sa population, une des solutions a été l'exode (Faouzi, 2003).

Le poids d'une croissance démographique est souvent cité. Cependant, l'effet de ce facteur est loin de faire l'unanimité. La vaste problématique des relations entre croissance démographique et environnement soulève de nombreuses controverses au sein de la communauté scientifique. De Malthus à Hardin ou de Condorcet à Boserup, pessimistes et optimistes se sont affrontés et s'affrontent encore aujourd'hui autour de la question de l'impact de la population sur l'environnement.

Toutefois, la plupart des positions suscitées par cette question de la croissance démographique, traduisent la position, la plus répandue qui fait référence à la thèse de Malthus (1798), selon laquelle, l'augmentation de la population entraîne un accroissement de la pression sur les ressources qui se traduit par une baisse de la fertilité de la terre, générant une diminution des rendements des cultures et donc une baisse de la production agricole disponible (Jouve, 2006). Nos observations sur le terrain montrent que certaines régions connaissent effectivement une évolution de type malthusien. Mais, on trouve aussi des régions où la forte densité de population n’a pas entraîné de dégradation.

\section{La croissance démographique : premier facteur majeur de l'évolution}

La population des Haha comptait 194004 habitants en 2014 répartis en 27316 ménages (Haut Commissariat au Plan, 2004), alors qu'elle ne comptait que 120300 habitants en 1960, enregistrant ainsi un taux moyen d'accroissement annuel de $2,8 \%$. Cela a eu pour conséquence une forte augmentation de la densité d'occupation à l'hectare qui est évaluée entre 13 à 15 habitants par hectare 
irrigué ce qui constitue une charge humaine très importante pour des ressources limitées.

Par le phénomène de la poussée démographique, le système traditionnel qui connaissait un relatif équilibre se trouve être perturbé par le manque d'espace disponible. En effet, au cours de son évolution, l'exploitation agricole familiale est confrontée à la nécessité d'ajuster ses ressources et ses besoins à mesure que sa taille augmente. Or, la densification de ces espaces, ajoutée à l'exploitation désordonnée et abusive de l'arganeraie, a fortement réduit les espaces forestiers «cultivables » et induit un phénomène de défrichement linéaire croissant de la forêt. La croissance démographique suscitant la course à la terre, l'on assiste aujourd'hui à une émergence des droits familiaux sur les terres cultivées. Ceux-ci sont de plus en plus marqués par une appropriation individuelle ou familiale des titres fonciers.

De l'exploitation de ces nouvelles terres forestières sont alors nés les conflits avec l'administration des Eaux et Forêts qui revendique la domanialité de tous les espaces forestiers. Certes, cette administration a permis de limiter les grignotages et les incursions des populations en forêt. Mais inversement, cet arrêt des extensions agricoles en forêt constitue « une nouvelle donne » et une nouvelle contrainte sociale et économique pour les populations. L'inadaptation et l'ancienneté des textes qui régissent l'utilisation des terres collectives ne facilitent pas, en outre, l'accès à ces terrains comme alternative de mise en valeur visant à soulager la forte pression sur l'arganeraie traditionnelle.

Dans la situation actuelle des Haha, la législation forestière n'est plus adaptée. Les paysans de la région n'ont, en fait, pas voix au chapitre en ce qui concerne la forêt domaniale, et la gestion qu'ils adoptent est coutumière et non écrite. Les conséquences de cet état de fait : une mauvaise gestion des terres cultivées sur l'espace domanial, l'absence d'entretien de l'arbre (arganier) après recépage, des tensions sociales et conflits de limites sur les terrains de plus en plus exigus et non clos, des actions répressives sur les délits de défrichement, etc.

Face à cette situation et à l'incapacité de l'activité agro-pastorale à satisfaire les exigences de la famille, l'adaptation économique a pris la forme d'une diversification des activités et d'une recherche d'opportunités de remplacement ou de compensation, en recourant au défrichement illégal de l'arganeraie.
Dans cette région où les potentialités d'extension des surfaces agricoles sont limitées, la pression démographique qui s'exerce sur la terre a pour corollaire l'atomisation parcellaire et le morcellement de la propriété par le jeu de l'héritage.

L'accroissement démographique qui, au cours de plusieurs décennies s'est accompagnée d'une incontestable intensification et qui par le jeu des divisions successorales, ne fait qu'accentuer le morcellement des terres et la précarité des propriétés agricoles. L'insuffisance des ressources qui se manifeste en premier lieu dans la part réduite des terres agricoles par habitant (figure 4), ne peut plus permettre à ces zones de retenir leurs populations, d'où les flux migratoires importants qu'elles connaissent.

\section{Émigration et fluctuations de l'espace cultivé}

Nombreux sont les travaux qui pointent du doigt les mauvaises conditions naturelles (sécheresse, manque d'eau, etc.) et l'insuffisance des ressources en terres comme étant les facteurs déterminants de l'émigration. La question qui se pose en effet est de savoir si l'intensité migratoire reflète la différenciation des modes d'accès aux ressources naturelles, et en particulier à la terre. Selon le modèle habituel de la pression foncière, on peut admettre que les individus ou les familles les plus démunis partent le plus. Autrement dit, la tendance à migrer serait liée à la taille de l'exploitation familiale. Malheureusement, nous ne disposons pas de chiffres fiables qui permettent de démontrer cette relation.

Ceux qui émigrent le plus sont ceux qui ont peu de terres (moins de deux hectares) et/ou qui n'ont pas accès à l'irrigation. La migration entraîne le prélèvement d'une main-d'œuvre potentiellement

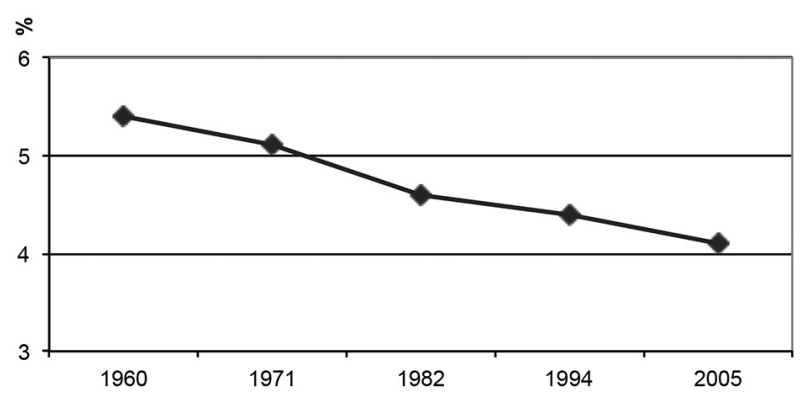

Figure 4 : Régression de la SAU en \% (source : DPA d'Essaouira, 2005) 
active, obligeant les femmes à prendre en charge la gestion de l'exploitation. Soumises à une gestion en solitaire de l'exploitation, les femmes ne parviennent pas toujours à optimiser les capacités productives de l'exploitation. Le poids des tâches agricoles, que les stratégies d'entraide (twiza) ne suffisent pas à compenser, entraîne souvent une sous-exploitation des ressources foncières disponibles. Les femmes, faute de moyens humains ou financiers, cessent de cultiver certaines parcelles qu'elles laissent alors à l'abandon. Ainsi, la féminisation de l'agriculture, corollaire de l'émigration, contribue à fragiliser les systèmes de production et à entraver la mise en valeur des terres.

Aujourd'hui, partout dans cet espace, on retrouve les traces d'anciens périmètres avec des aménagements hydrauliques, qui par le passé ont été irrigués et cultivés. Interrogés à propos de ces anciens espaces irrigués aujourd'hui disparus, les hihis surtout les hommes âgés de la région avancent les deux causes suivantes : sécheresse et émigration.

Donc, deux facteurs principaux se conjuguent pour expliquer les causes précises de ces disparitions et l'amenuisement de surfaces irriguées dans la région. Il s'agit d'une part de la sécheresse chronique combinée au manque d'eau chronique et l'ensablement de certaines arganeraies réduisant ainsi la superficie des terres déjà exploitées, et d'autre part de l'émigration qui a privé l'agriculteur de la maind'œuvre masculine. Dans le même temps, l'importance des revenus rapatriés a démotivé la famille pour le travail agricole. Le manque de main-d'œuvre est particulièrement inquiétant, surtout au moment des gros travaux agricoles. On comprend, comme le note un rapport de la Direction Provinciale d'Agriculture d'Essaouira 2001, pourquoi «la femme rurale est très impliquée dans les activités agricoles». Elles ne peuvent cependant s'occuper de l'aménagement et de l'entretien des champs et des seguias ainsi que des travaux d'irrigation. Cela a des répercussions sur les disponibilités en eau. Dans notre région, l'émigration touche environ $34 \%$ de la population, dont $32,5 \%$ d'hommes et $1,5 \%$ de femmes (Faouzi, 2003).

Chez les Ait Zelten, dans la partie nord-est du territoire des Haha, l'émigration touche environ $34 \%$ de la population. Sur les $66 \%$ restants, seuls $10 \%$ sont agriculteurs (HCP, 2016). Les facteurs qui contribuent à alimenter ce flux sont sans doute si complexes que nous ne prétendons guère pouvoir les énumérer et les éclaircir. Le facteur démographique n'explique pas tout. C'est ainsi que la part du facteur «assiette foncière » est bien nette dans l'alimentation du flux migratoire : le nombre d'émigrés augmente avec, entre autres, la taille de la famille et la réduction des terres agricoles en sa possession.

Dans la région, l'émigration n'a pas abouti à la concentration de la propriété chez les familles de migrants. C'est plutôt l'inverse qui s'est produit, car l'argent gagné à l'étranger a rendu le travail de la terre et toutes les activités qui y sont liées moins nécessaires. Dans les communes de forte migration, les terres qui étaient exploitées autrefois restent actuellement incultes. Il en est de même des petites surfaces irriguées à l'aide des "seguias ». La généralisation de l'émigration, l'importance des revenus migratoires, le développement de l'émigration familiale, l'aggravation de l'exode rural vers les villes et l'apparition d'activités non agricoles ont participé à la réduction des espaces irrigués. La monétarisation de l'économie locale a incité la masse des hihis, dont la situation ne cesse de s'aggraver, à rechercher hors de l'agriculture d'autres sources de revenus. Ainsi le retour annuel des émigrés, leurs nouveaux moyens économiques (voitures, liquidités, construction d'une nouvelle maison...), ont encouragé ceux qui travaillaient encore au bled à partir à leur tour. Ces départs supplémentaires privent ainsi les douars d'une partie de leur main-d'œuvre masculine nécessaire pour assurer les gros travaux de la terre, surtout pendant les périodes de pointe (labours, semailles, récoltes), et contribuent à faire baisser la productivité.

Ces fluctuations de l'espace irrigué ne signifient pas cependant l'irrésistible disparition de la vie dans l'arganeraie des Haha. Partout dans la région, on assiste à l'extension des mises en culture. Depuis les années 1970, les arbres sont systématiquement éliminés pour favoriser le passage de la charrue (figure 5).

Sous l'influence du droit musulman et des conceptions occidentales introduites par la colonisation, « les concessions foncières coutumières ont été assimilées progressivement à des propriétés privées » (Fay, 1987, p. 243). Conséquence de cette pénétration de structure socio-juridique : les mises en culture et plantation d'arbres fruitiers, réputées comme des signes indiscutables d'appropriation, se sont développées partout où l'araire pouvait entrer. 


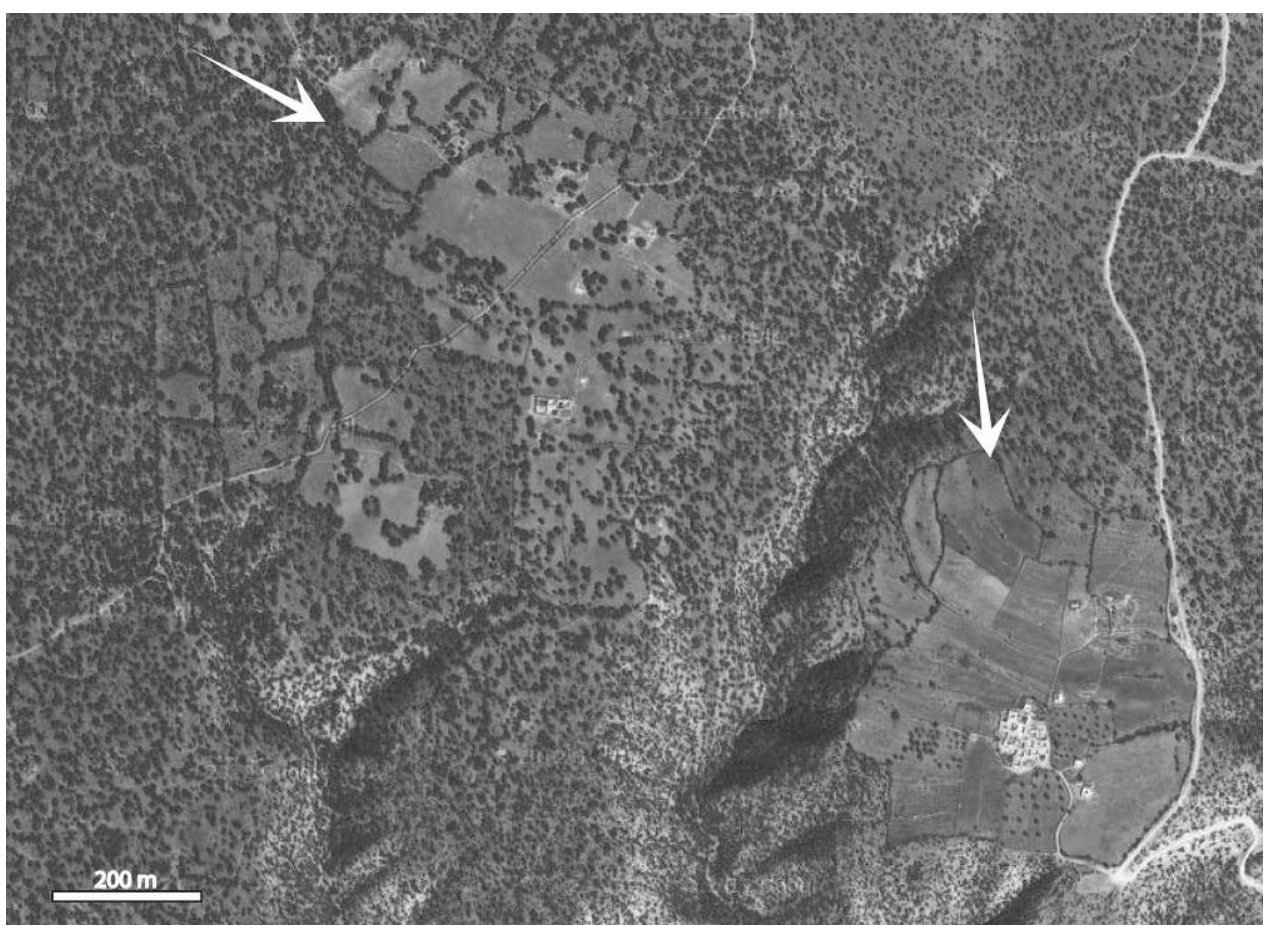

Figure 5: Vue aérienne des clairières au nord-ouest de Tamanar (douar Ouchene) Cet exemple illustre bien le défrichement des arganeraies pour étendre les terres cultivables. Tous les témoignages concordent pour dire que dans les années 1970, une véritable forêt et une végétation étaient très denses à cet endroit (source : CNES/ SPOT, 2013) Aerial view of clearings on the north-west of Tamanar (douar Ouchene) This example well illustrates the clearing of Argan treein in order to extend cultivable lands. All the testimonies match to say that in the 1970 s, a real forest and a vegetation were very dense in this place.

Avec l'accroissement démographique le processus d'extension des superficies cultivées va s'accentuer. Partout, on observe une extension des superficies défrichées et mises en culture. Les propriétaires procèdent à l'élargissement de leurs champs aux dépens des friches voisines de statut théoriquement collectif ou domanial. Bien souvent, « les défrichements progressent à partir des fonds de vallée en remontant vers les crêtes ou rebords de plateaux, sur des pentes trop fortes. Les rendements diminuant, les fellahs iront défricher de nouvelles terres mais les parcelles qu'ils ont défrichées ne seront pas pour autant reconquises par le matorral ou la forêt puisque les troupeaux continueront d'y chercher pâture. On est bien ici en présence d'une agriculture minière effectuée aux dépens des terrains collectifs ou présumés domaniaux. [...] En effet, les premières délimitations ont déclenché une course aux défrichements et à la mélkisation puisque l'éradication de la végétation ligneuse privait les forestiers de toute justification pour domanialiser le sol » (Fay, 1987, p. 238) (figure 6 - planche V).

Sous l'effet de la pression démographique, de la faible disponibilité en terres agricoles et en eau d'irrigation, cet espace est devenu incapable de nourrir la population. C'est ainsi que les familles sont tombées dans un état de crise chronique, obligeant une partie de la population à émigrer vers d'autres régions. Pour ce dernier facteur on peut procéder à deux remarques contradictoires : si l'émigration a participé à l'amenuisement de surfaces cultivées, elle va parallèlement accentuer encore plus le processus d'extension des terres cultivées et l'intensification agricole par l'investissement en capital, c'està-dire l'acquisition de matériels, la construction de bâtiments, l'utilisation d'intrants et la réalisation d'aménagements.

Deux tendances contraires apparaissent : d'un côté, une déprise agricole et, de l'autre, une intensification des systèmes de production et une valorisation de la terre. En fait, pour les migrants qui parviennent à épargner, l'achat de terres constitue l'autre moyen d'accès à la propriété. Les transactions foncières sont très dynamiques dans cette région : la terre est rare et très chère. Toutefois, elle continue d'être achetée.

Dans les années 1980 et 1990, les investissements des émigrés, en particulier les anciens khammès (métayers au 1/5) ou rabaas (métayer au 1/4) s'orientaient davantage vers le secteur agricole, surtout dans l'achat de terres agricoles qu'ils ont équipées de matériels sophistiqués (tracteur, motopompe, véhicule de transport, etc.).

Partout chez les Haha, l'utilisation des tracteurs et des batteuses gagne de plus en plus de terrain. 


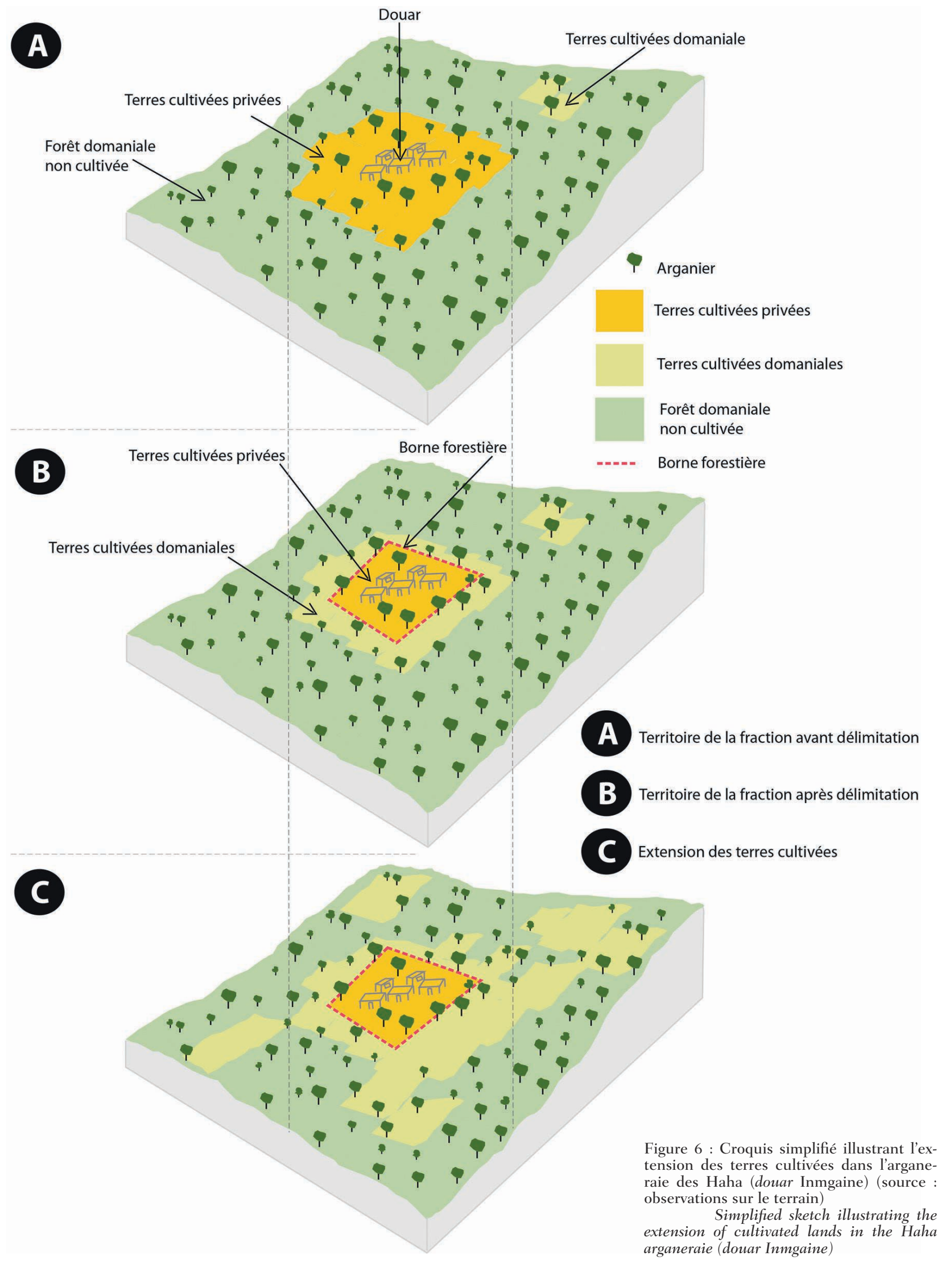



L'utilisation des tracteurs pour les labours ne cesse d'augmenter. Le recours à la mécanisation permet de gagner du temps : auparavant pour retourner un hectare, il fallait au moins une dizaine de jours. En outre, la désaffection pour les travaux agricoles et l'émigration ont poussé les fellahs à faire usage de ces engins. Cette mécanisation qui évolue lentement mais qui ne s'est pas substituée entièrement aux travaux manuels, est née d'une situation nouvelle, notamment l'abandon des travaux agricoles par les jeunes.

En plus de la mécanisation, les fellahs utilisent de plus en plus les engrais dès que leurs moyens financiers le permettent. Ces engrais sont utilisés généralement pour toutes les cultures. Ainsi dans certaines régions, ils ont développé une agriculture capitaliste rompant avec tous les systèmes traditionnels.

Pendant des siècles, l'irrigation traditionnelle était assurée grâce à la dérivation des eaux superficielles et par prélèvement des eaux souterraines grâce à des puits à l'aide de la traction animale (dlou). À partir des années 1970 et 1980, la population a développé une mise en valeur agricole intensive et l'irrigation moderne a commencé dès lors à prendre place dans la région, d'où la nécessité de creuser des puits profonds et d'installer des motopompes. En 1991, la seule commune de Smimou comptait déjà 39 motopompes, 5 moissonneuses modernes, 10 pulvérisateurs. De même, tout au long des oneds de la région des Haha les parcelles cultivées sont irriguées par motopompes (figure 7).

Jusqu'aux années 1970, le nombre des motopompes était très limité. C'est à partir des années 1980-1990 que leur nombre devient plus important, entraînant ainsi une série de changements lisibles sur l'espace. L'expansion de ces pratiques a eu pour conséquences un abaissement très spectaculaire du niveau de la nappe.

L'étude du niveau de la nappe phréatique dans les puits de la région des Haha montre un rabattement très spectaculaire du niveau de la nappe. Selon les chiffres de l'agence du bassin hydraulique du Tensift concernant l'état du niveau de la nappe phréatique, cette dernière a baissé de $65 \mathrm{~m}$ dans les années 1970 à plus de 400 m dans les années 2000 pour les communes de Smimou et de Tamanar.

Partout, à côté des puits, on voit des traces de creusement récent; signes d'essais nouveaux pour approfondir les puits et indices d'un mauvais approvisionnement en eaux souterraines. De tels investissements montrent que l'agriculture irriguée n'est qu'une question de survie dans cette région. Nombreuses sont les exploitations qui ont complètement dépéri et ont été totalement abandonnées à cause du manque d'eau (figure 8).

Plusieurs exploitations actuellement mises en valeur, souffrent d'une pénurie en eau immense.

\author{
Figure 7 : Parcelles \\ irriguées dans les douars \\ d'Agadir Isserdane, Zaouit \\ Issouquine, Azaarad \\ (source : CNES/SPOT, \\ août 2015) \\ Irrigated Plots \\ in the douars of Agadir \\ Isserdane, Zaouit Issou- \\ quine, Azaarad
}

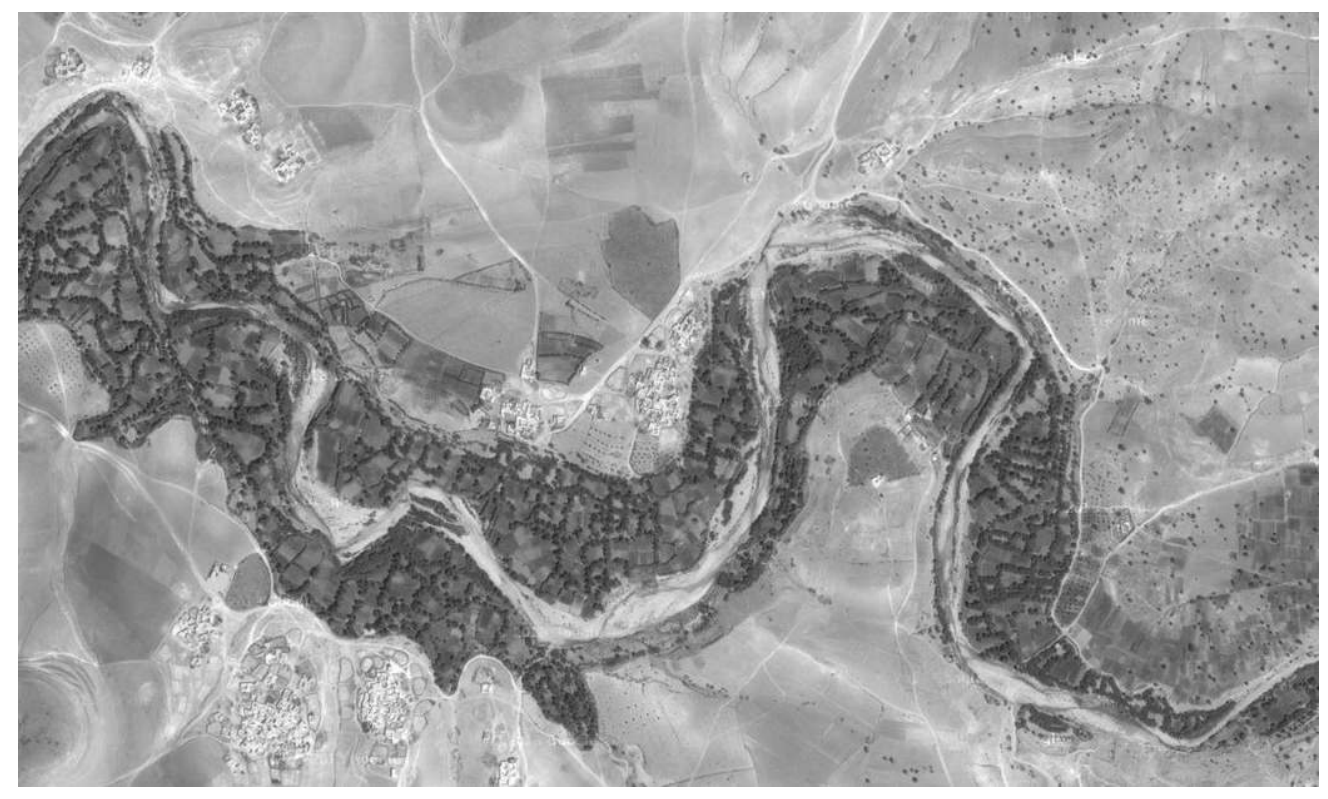




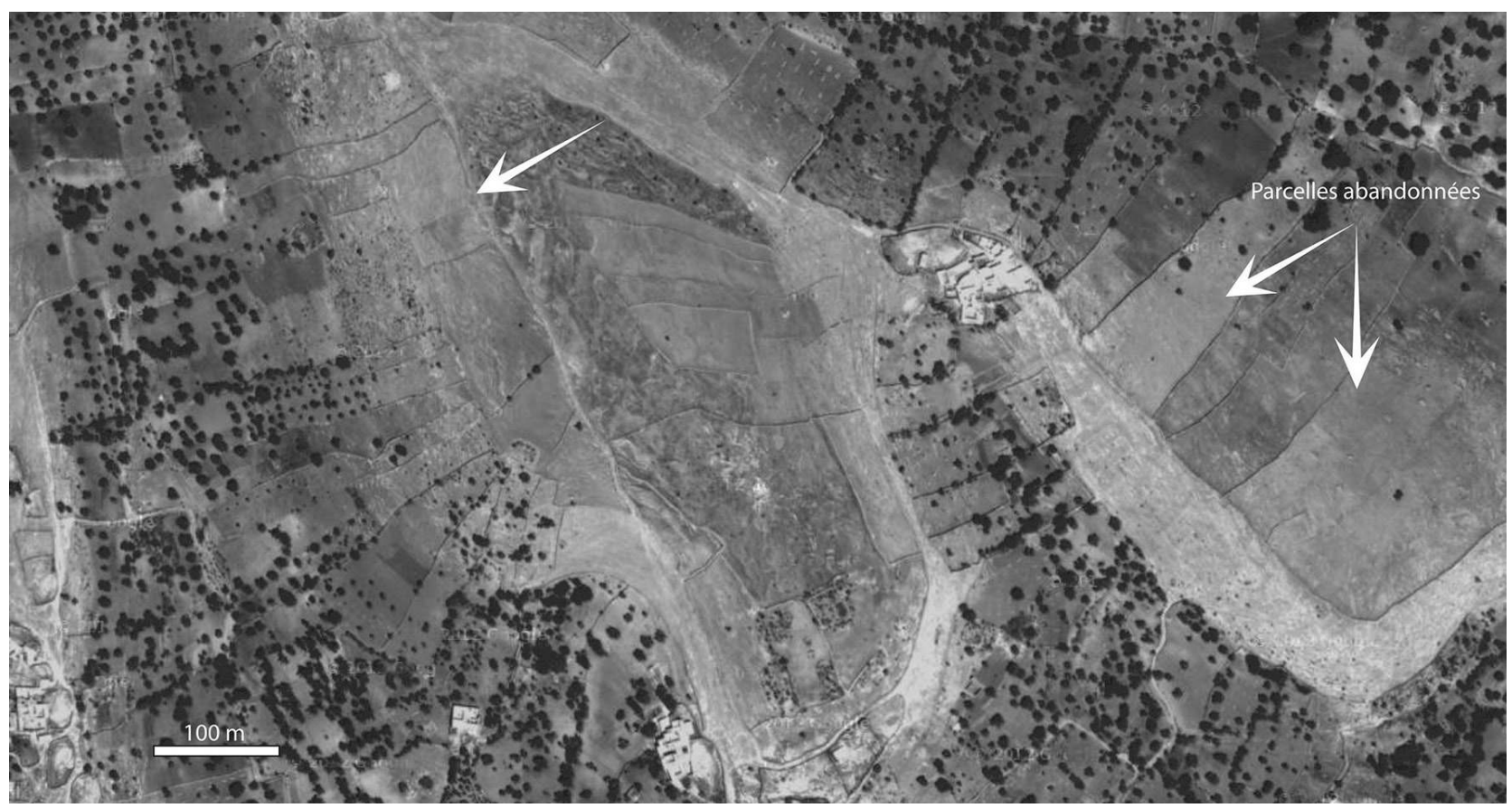

Figure 8 : Déprise agraire : sur la photo satellite du douar Imi Ntlit, on voit les traces d'exploitations qui ont été totalement abandonnées à cause du manque d'eau. Ces terres portent des traces d'érosion hydrique (source : CNES/SPOT, 2013)

Agrarian abandonment: on the satellite photo of the region of Imi Ntlit, we see the tracks of exploitations (operations) which were totally abandoned because of the lack of water. These lands carry tracks of hydric erosion

Ces parcelles sont les plus menacées de tomber en friche. Chaque année, de nouvelles parcelles sont abandonnées et les productions baissent constamment. Ce phénomène de pénurie en eau touche pratiquement toutes les exploitations et la situation est précaire dans toutes les fractions des Haha.

Comme les disponibilités en terre ne sont pas illimitées, le développement d'une agriculture, qui satisfasse aux besoins d'une population en forte croissance, nécessite une intensification de la productivité de la terre. Pour cela, les agriculteurs sont amenés à allonger leur temps de culture et à raccourcir la durée de la jachère ce qui est insuffisant pour restaurer la fertilité du sol. Il en résulte une diminution de la productivité de la terre, obligeant ainsi les agriculteurs à abandonner leurs champs (Jouve, 2006). C'est dans ce type de situation que l'on a pu observer des mutations agraires de type malthusien avec une diminution progressive des rendements des cultures (Jouve, 2006).

Après avoir connu des siècles d'abondance, à en croire le géographe arabe Léon l'Africain (14881548) qui, dans son ouvrage "Description de l'Afrique ", décrivait le pays des Haha comme une région très prospère, cette dernière connaît actuel- lement une situation très précaire. Avec les apports de l'émigration, la région des Haha se trouve en pleine évolution. Si ces mutations se manifestent dans l'espace par les changements du paysage, les modes d'exploitation et le comportement des gens n’en restent pas moins touchés.

\section{Des modes d'exploitation et un mode de vie et en voie de disparition}

L'injection massive des revenus, grâce à l'émigration, a conduit la monétarisation des rapports sociaux et fait en quelque sorte disparaître les anciens modes d'exploitation et les anciennes formes de solidarité et d'entraide au sein de la société hihi.

Ainsi le khammès et le rbâa qui travaillaient contre le cinquième et le quart de la récolte, tendent à disparaître, du fait que la population agricole n'accepte plus ces formes dont les revenus faibles et aléatoires ne répondent plus aux besoins du métayer.

Devant la disparition du khamès et du rbâa, la désaffection des jeunes pour le travail agricole et la dévalorisation de certains métiers, les propriétaires font de plus en plus appel au travail salarié. 
Jusqu'à un passé récent, le paiement du berger se faisait en nature selon le contrat conclu avec le propriétaire, mais aujourd'hui il préfère être payé en argent. C'est ainsi que cette région durement touchée par la pauvreté, voit émerger une nouvelle catégorie sociale, celle d'agriculteurs temporairement salariés et pluriactifs.

Quoi qu'il en soit, le recours à la main-d'œuvre salariée est un phénomène lié à l'évolution de la société hihi où la twiza entre les membres de la communauté a disparu, où la femme travaille rarement la terre et s'occupe essentiellement de la maison et de l'éducation des enfants. Avec l'émigration les habitants se libèrent des traditions et leur mentalité change, l'émigré accepte facilement que ses filles aillent à l'école. Si cette scolarisation a favorisé l'épanouissement des jeunes scolarisés, elle a par ailleurs privé l'activité agricole d'une main-d'œuvre enfantine largement exploitée chez les Haha. Ainsi, l'injection massive des revenus migratoires a contribué à faire disparaître les vieux usages communautaires et à développer le salariat; il s'ensuit la disparition progressive des valeurs traditionnelles comme la twiza (l'entraide) qui faisaient la cohésion du groupe. À cela s'ajoute un autre aspect de ces conséquences sociales. Sous l'action de l'évolution économique, la société hihi est devenue de moins en moins homogène et tend à être de plus en plus hiérarchisée.

Les bouleversements sociaux ont surtout engendré le démantèlement des structures familiales et l'éclatement de la grande famille traditionnelle avec comme corollaire le partage des biens et les ruptures d'indivision, qui créent autant de nouvelles exploitations qu'il y a d'héritiers, entraînant ainsi la création d'une multitude de micro-exploitations.

L'extension considérable de la micropropriété (figure 9) pose le problème de la modernisation agricole, et ne peut ni nourrir ni occuper les actifs des ménages agricoles. Le partage du patrimoine entre les héritiers, autrefois l'exception, est aujourd'hui la règle. Il conduit les héritiers à s'installer sur des exploitations de plus en plus petites.

À l'échelle du douar (village), de la fraction et parfois même de la tribu, l'émigration a créé de véritables disparités entre les foyers des émigrants et ceux des non-émigrants. Les revenus migratoires ont joué le rôle décisif dans l'ascension sociale des familles qui étaient jusqu'à une date récente au bas de l'échelle sociale. Actuellement, l'émigré est considéré comme faisant partie d'une fraction sociale se situant au niveau le plus élevé de la hiérarchie « une classe sociale nouvelle apparait donc, dont les modes de vie tendent sur ceux de la ville» (Robine [1956], cité par Bellaoui, 1986, p. 226). Ainsi, à l'élite traditionnelle (descendants des anciens chioukh et caïds, ou des chefs des zaouias, etc.), se substitue une nouvelle élite formée de commerçants et travailleurs émigrés.

La monétarisation de l'économie villageoise a aussi obligé les plus pauvres à disposer à leur tour de
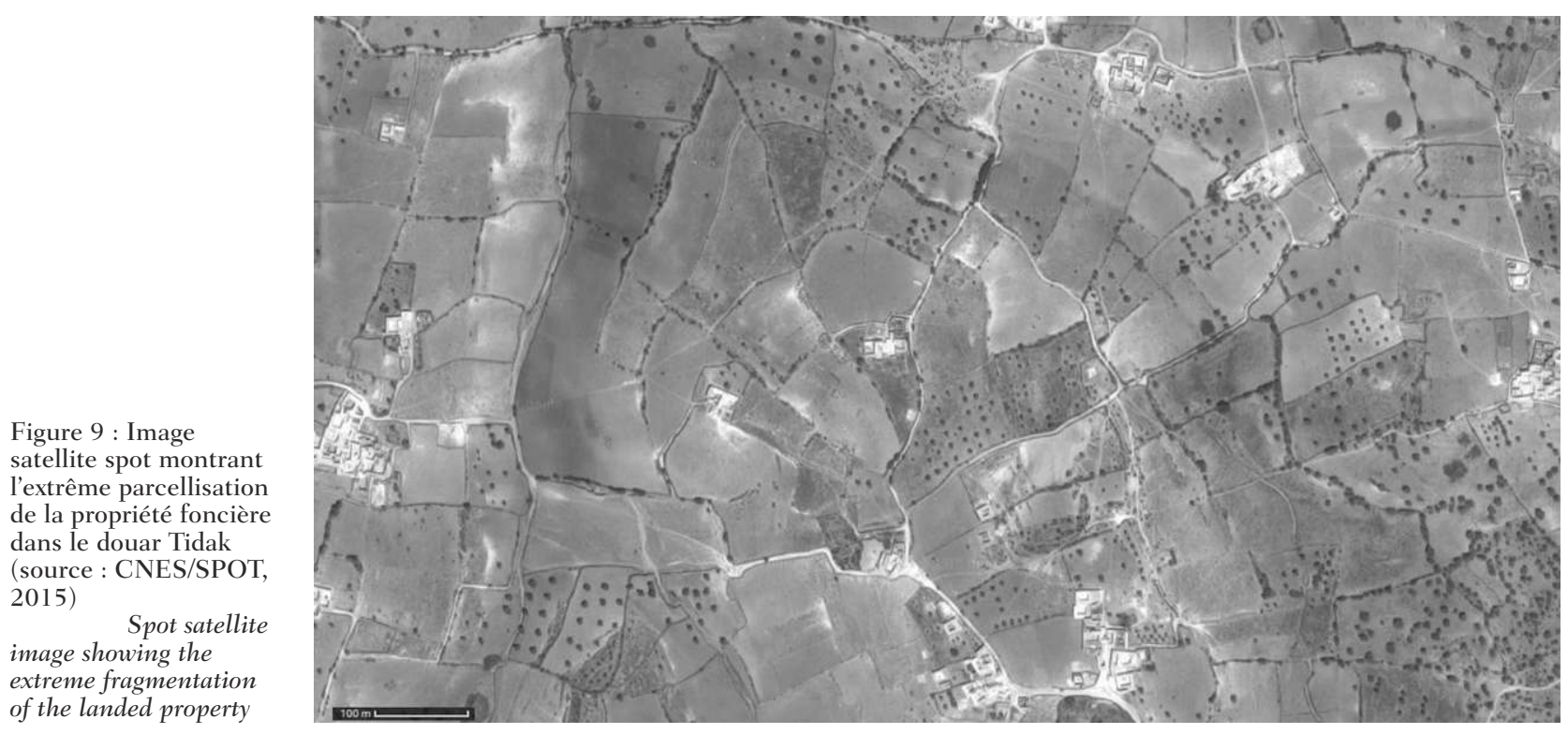
revenus qu'ils se procurent par le commerce de bois récoltés dans la forêt d'arganiers surtout dans les espaces boisés domaniaux. Partout chez les Haha, des convois de mulets chargés de bois de chauffage (au prix moyen de 25 dirhams la charge) ou de sacs de charbon de bois récoltés en forêt, circulent, le plus souvent la nuit, pour alimenter les souks locaux.

Nous avons vu que l'émigration dans la région est une réponse directe au déséquilibre entre une population, sans cesse croissante, et des ressources de plus en plus limitées. Cette émigration est un des moyens de rétablissement de cet équilibre en raison des importants revenus qu'elles drainent sur les milieux de départ.

\section{Conséquences de l'émigration sur l'économie locale}

Les revenus de cette émigration servent, par sens du devoir et de solidarité familiale, à assurer les dépenses de la famille restée sur place, entraînant ainsi, un profond changement de l'économie locale.

D'après nos enquêtes menées sur le terrain de 1996 à 2011, environ $33 \%$ de la population touchée par l'émigration ont déclaré que les ressources de l'émigration constituent la seule source de revenu familial. Tandis qu'environ $67 \%$ ont déclaré que les revenus migratoires représentent plus de $50 \%$ de l'ensemble des ressources de la famille.

Cet apport considérable de fonds liés à l'émigration revêt un caractère vital dans l'économie domestique. La majorité des partants des Haha préfèrent utiliser leurs gros revenus dans la pierre (construction et l'amélioration de locaux d'habitation), engendrant de profondes transformations très visibles dans l'espace, métamorphosant ainsi les paysages des Haha.

\section{Un habitat mal adapté au genre de vie traditionnel}

L'habitat rural subit des transformations, aussi bien sur le plan architectural que dans l'usage des matériaux de construction. Ces transformations ont eu pour conséquence un habitat peu ou mal adapté au climat et au genre de vie traditionnel.

En effet, l'évolution démographique, l'émigration, les changements des modes de vie et l'ouverture de la région sur l'économie extérieure ont imposé une évolution architecturale, spatiale et fonctionnelle de l'habitat. Ces mutations échappent de plus en plus au contrôle collectif. Les initiatives privées quant à elles se multiplient et les mutations prolifèrent.

L'éclatement des douars et l'apparition de l'habitat individuel, l'abandon des techniques anciennes ainsi que la nouvelle organisation interne de la maison en témoignent. Les techniques architecturales anciennes, pierres et pisé, cèdent la place d'une manière rapide aux techniques contemporaines à base de béton armé et d'agglomérés industriels (figure 10). Les pièces sont de plus en plus larges et pourvues de multiples fenêtres de grandes tailles. Elles sont souvent situées à l'étage. Quant au rezde-chaussée, celui-ci abrite parfois des magasins fermés.

Ces nouvelles constructions sont mal adaptées au milieu naturel caractérisé par la rigueur du climat. Le béton armé, l'épaisseur très réduite des murs et les grandes dimensions des fenêtres ne permettent plus une isolation parfaite de la maison, celle-ci se transforme en un véritable four en été. Cette architecture moderne, sans caractère, choque les quelques visiteurs (touristes, chercheurs) de passage dans cette région.

Parallèlement à ces changements, l'organisation fonctionnelle de la maison s'est modifiée pour répondre aux nouveaux besoins de la population. Ces changements ont commencé par l'éloignement de l'étable de la demeure. La cour se transforme en un petit jardin, la tamsrieyt (salon réservé aux invités) prend une grande place dans l'espace bâti. La

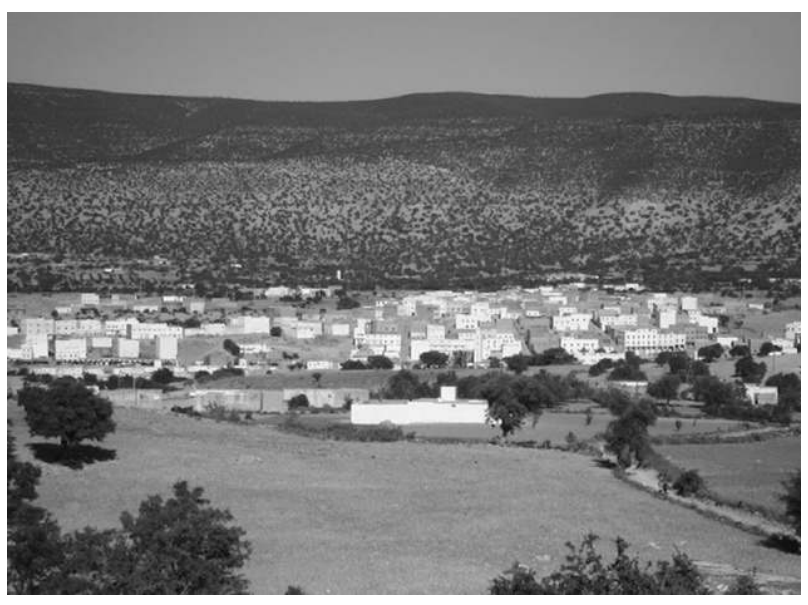

Figure 10 : L'apparition d'un habitat de type moderne dans le douar Asayiss

The appearance of a modern housing in the douar Asayiss 
cuisine fait son apparition ainsi que la salle de bain et les toilettes.

Ces transformations se manifestent aussi par une évolution interne de la maison touchant essentiellement le mobilier. L'évolution relative du niveau de vie a renforcé chez le paysan le désir de se meubler de façon moderne pour adopter un environnement domestique largement influencé par la vie citadine. Le four traditionnel utilisant le bois combustible perd progressivement sa place en faveur du four à gaz. Les ustensiles de cuisine qui ont été dans leur majorité en bois et en poterie sont remplacés par des produits industriels.

\section{L'arganeraie des Haha face à une charge pastorale importante}

L'arganeraie des Haha est un espace riche aux potentialités de pâturage, il est probable même qu'il soit la première activité qu'a exercée la population locale. Chez les Haha, le cheptel tient une place importante, d'autant plus importante que les hihis voient en l'élevage des caprins et des ovins une expression de richesse.

En 1982, selon la Direction Provinciale de l'Agriculture d'Essaouira (2003), ces troupeaux comptaient 120000 ovins, 170000 caprins et 15000 camelins. L'avènement de l'émigration va occasionner une charge pastorale très importante. En fait, à partir de capitaux rapatriés se sont constitués de grands troupeaux composés surtout de caprins et de moutons dont la valeur commerciale est plus grande et gérés en association avec le recours à des bergers du douar. Ainsi, aux troupeaux familiaux traditionnels, se sont progressivement ajoutés ces nouveaux venus. Nous ne disposons malheureusement pas de données plus récentes. Toutefois, selon les chiffres de la Direction Provinciale de l'Agricul- ture d'Essaouira datant de 2011, ces effectifs se sont multipliés par trois (tableau 1 et figure 11).

Ces statistiques sont d'ailleurs discutables car le nomadisme amène des troupeaux de régions parfois éloignées (régions sahariennes du Maroc), à la recherche de l'aâcbeb (herbe). C'est le cas des troupeaux de dromadaires que l'on déplace sur de grandes distances, ce qui participe à la dégradation de l'arganeraie.

Chez les Haha, l'élevage est conduit de façon extensive et basé presque exclusivement sur l'utilisation des parcours hors et sous-forêt d'arganier. Cet accroissement du cheptel va entraîner une forte pression sur les ressources, surtout dans les zones où les peuplements sont plus clairsemés, sous l'effet d'un processus de désertification ayant pour conséquence directe une dédensification des peuplements (Naggar et Mhirit, 2006).

Partout dans la région, par suite d'une augmentation rapide de la population, on assiste à une extension des terres de culture et à la multiplication des agdals au détriment des terres de parcours.

En fait, la parcellisation extrême au fil des successions et des partages a introduit dans le terroir de plus en plus de cas d'agdals inaccessibles. De plus, les cas d'indivision se multipliant, bon nombre d'agdals ne sont plus autorisés au pâturage pendant la période de mise en défens. Conséquence immédiate, le surpâturage des mouchaâs collectifs s'aggrave (figure 12). Ces derniers présentent des

\begin{tabular}{|l|c|c|c|c|}
\hline & Caprins & Ovins & Bovins & Camelins \\
\hline Nombre de têtes & 379100 & 415866 & 49651 & 8000 \\
\hline
\end{tabular}

Tableau 1 : Effectif du cheptel chez les Haha (Source : DPA., Essaouira, 2011)

Number of livestock in Haha
Figure 11 : Évolution de l'effectif du cheptel chez les Haha (source : d'après la DPA, Essaouira, 2011) Evolution of the size of the livestock in Haha

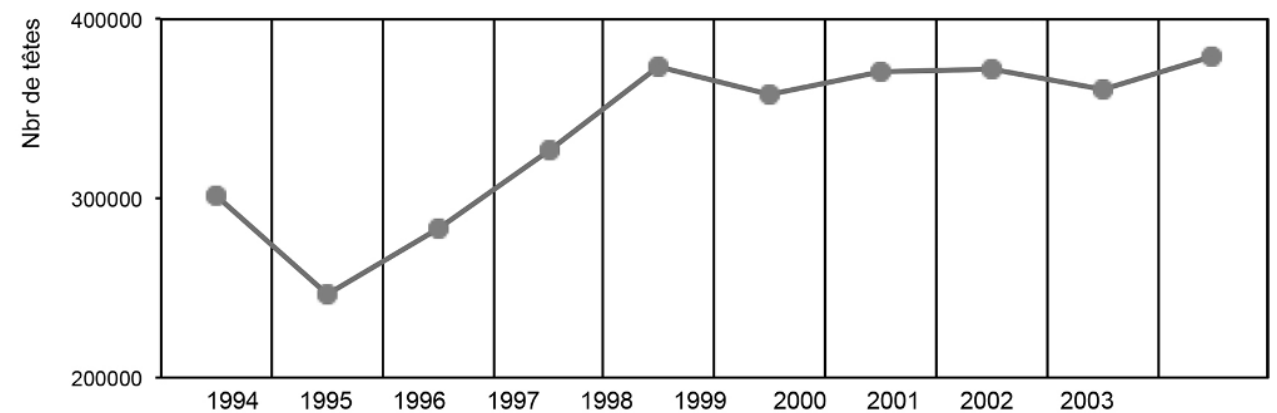




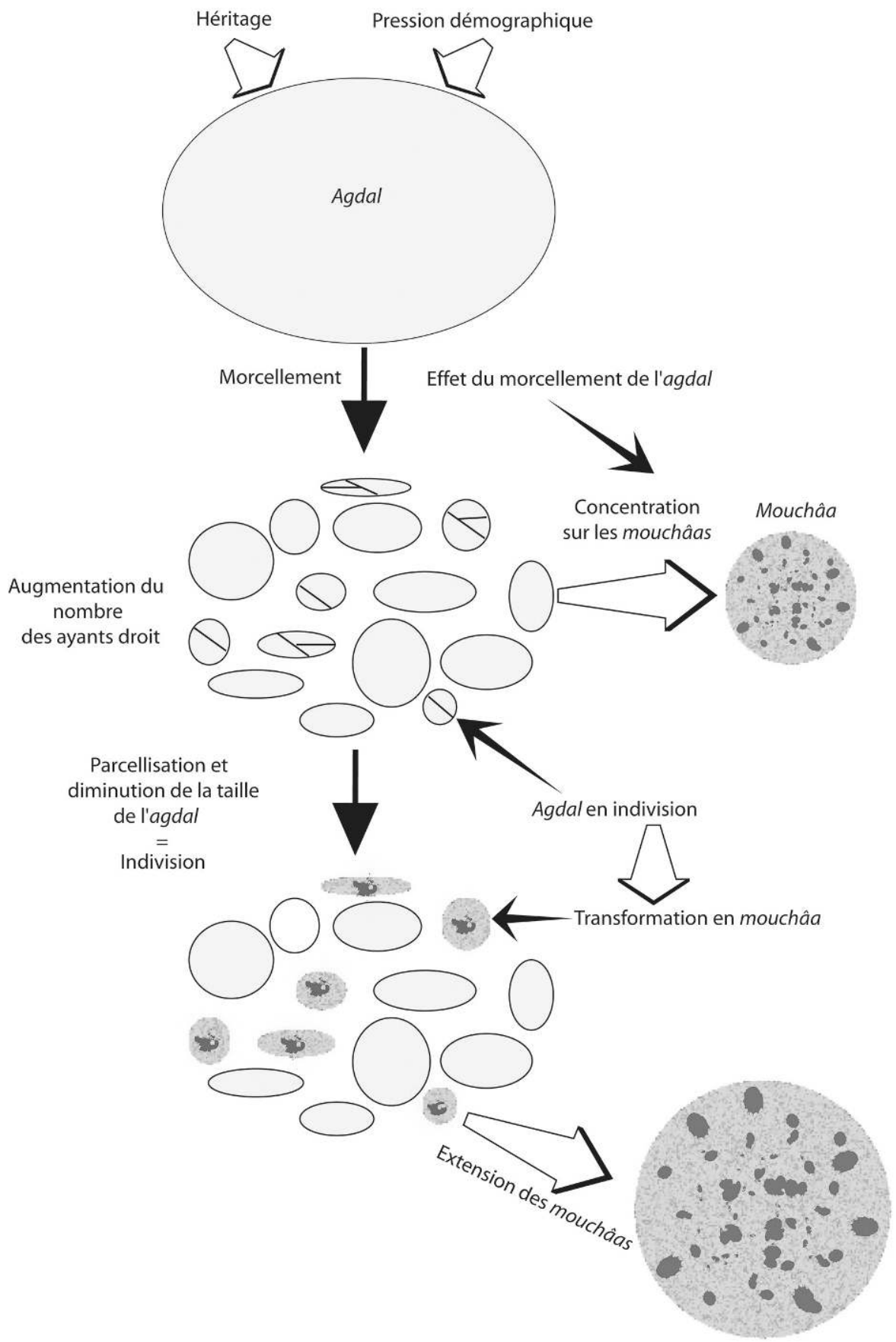

Figure 12 : Schéma illustrant l'impact de la croissance démographique et des systèmes d'héritage sur l'arganeraie (source : Faouzi, 2011)

Plan illustrating the impact of the population growth and the systems of inheritance on the arganeraie traces de surpâturage, les arganiers y sont sous forme de buissons ou de petits arbres en diabolo. En réalité, le fait de laisser les chèvres (taghate) en pacages libre pendant plusieurs mois est particulièrement redoutable, les caprins (figure 13) sont les animaux les plus nuisibles de tous et qui participent le plus activement à la dégradation des sols (destruction de nombre d'espèces arbustives et herbacées, écrasement des semis, consommation des graines) (figure 14).

D'après nos observations sur le terrain, on note l'apparition d'espèces vénéneuses et épineuses (Ziziphus lotus et Onanis natrix) dues au pâturage. La présence, de l'une ou l'autre de ces plantes indique une pression anthropozoogène très forte et la disparition des espèces caractéristiques des asso- 


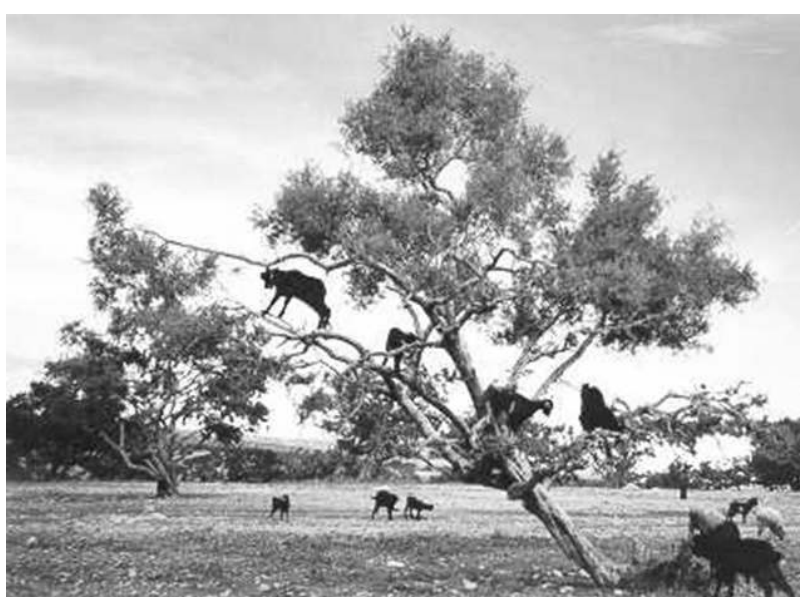

Figure 13 : Targant n'taghat ou l'arganier de la chèvre

Lutilité la plus quotidienne de l'arganier réside incontestablement dans son feuillage, véritable pâturage suspendu, consommé par les milliers de chèvres qui grimpent jusque dans sa cime. Au pâturage aérien, s'ajoute le pacage du tapis herbacé proprement dit et des chaumes après la récolte des céréales (cliché H. Faouzi, 2003). Targant n'taghat or goat argan

The most useful daily argan undoubtedly resides in its foliage, true suspended pasture, consumed by thousands of goats that climb into the top. To grazing air is added the grazing of the grass specifically and stubble after the grain harvest.

ciations forestières avec l'arganier, indiquant ainsi un bouleversement complet de la composition floristique de l'arganeraie.

Si la charge du bétail qui pâture l'arganeraie est élevée, néanmoins, ces dernières années on assiste à une réduction du cheptel caprin au profit des bovins et ovins pour diverses raisons.

\section{L'élevage caprin, l'activité la mieux adaptée est en régression}

La situation actuelle de l'élevage caprin chez les Haha connaît un retard net de développement par rapport à l'élevage bovin ou ovin. Ces modifications dans la composition du cheptel rejoignent les variations intervenues dans la gestion de l'arganeraie. Les terres de parcours collectifs tendent à régresser en raison de la parcellisation extrême au fil des successions et des partages ce qui a introduit dans le terroir de plus en plus de cas d'agdals inaccessibles.

La chèvre a toujours été considérée par les forestiers comme une espèce dévastatrice des peuplements forestiers et arboricoles; à ce titre elle est souvent taxée d'ennemie de l'environnement, et son accès aux parcours restreint l'expose à des pénuries alimentaires et la prive de ressources fourragères

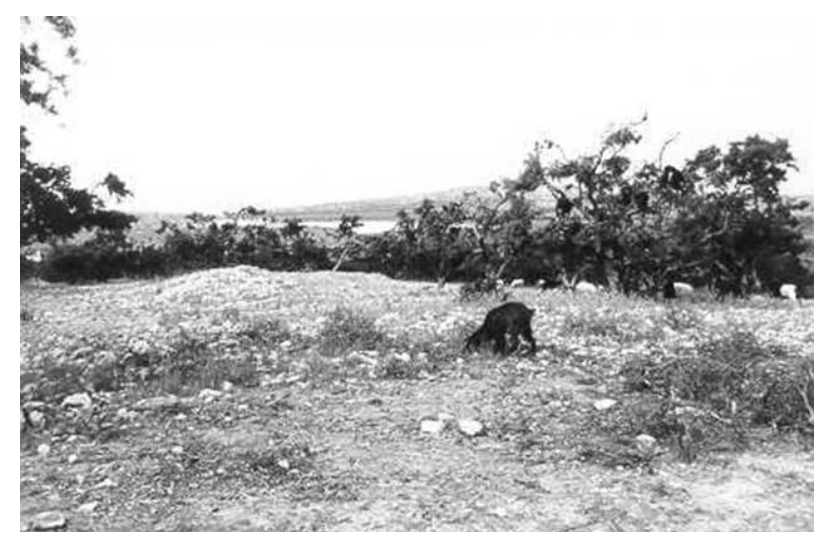

Figure 14 : Forêt dégradée (mouchâa, plateau des Ida Ou Throumma)

Exemple d'arganeraie-forêt dégradée dans la région des Aït Daoud. Signe d'une dégradation récente. Partout on traverse ce genre de formations effrangées et trouées d'arbres sévèrement ébranchés (cliché H. Faouzi, 2003).

Throumma)

Degraded forest (mouchâa, platean of Ida Ou

Example of argan-degraded forest in the region of Ait Daoud. Signs of recent deterioration. Everywhere we go, we cross this kind of unthreaded and pierced with holes formations of severely delimbed trees.

appréciables. La répartition des caprins dans les zones défavorisées a sérieusement limité les possibilités de développement de cet élevage.

D'autres paramètres importants font que l'élevage caprin perd en mobilité, entre autres, la « législation anti-chèvre » entrave la reconstitution du cheptel caprin et l'extension des surfaces cultivées qui s'étendent au rythme de l'accroissement démographique. Donc la qualité des parcours diminue, car ce sont les meilleures terres qui sont cultivées. Ainsi la chèvre, l'espèce la mieux adaptée au parcours extensif, cède la place aux ovins qui se prêtent plus à un élevage semi-intensif. Face à ces conditions défavorables pour la pratique de l'élevage, d'anciens éleveurs ont choisi l'adoption de l'agriculture et de s'adonner à d'autres activités plus rémunératrices comme la vente de l'huile d'argan.

\section{LES SYMPTÔMES DE DÉSÉQUILIBRE DE CET ÉCOSYSTÈME}

L'arganeraie des Haha souffre de beaucoup de problèmes, notamment le surpâturage, l'extension des cultures et les prélèvements en bois de feu (Faouzi, 2013) qui risquent de compromettre sa pérennité. Cette arganeraie connaît depuis des années une pression humaine souvent génératrice de problèmes. Les familles dans la région sont de grande taille, ce 
qui se traduit par une demande accrue de besoins. Ces besoins ne peuvent être satisfaits qu'à partir de la forêt d'arganiers. Le système actuel est incapable d'assumer la montée démographique avec son corollaire de besoins croissants autrement que par la paupérisation ou l'expulsion, c'est-à-dire la nécessité d'aller chercher à l'extérieur d'autres ressources.

En effet la croissance démographique se traduit par l'accroissement des besoins de la population en terrains de culture et d'habitat, en bois de feu pour la satisfaction des besoins domestiques des riverains de la forêt et aussi en fourrage pour l'alimentation du cheptel. Cette pression est d'autant plus accentuée que le niveau de développement des zones concernées est limité et le revenu des populations est très faible.

Le pâturage dans l'arganeraie connaît quant à lui une extrême dégradation. La multiplication des troupeaux caprins qui pratiquent pour la plupart un pâturage aérien des arganiers tout au long de l'année, engendre une surexploitation pastorale des peuplements arganiers, qui s'amplifie lors des années de sécheresse.

L'adoption de l'élevage extensif par les hihi (surtout les paysans ne possédant ni arganiers ni terre), soulève la question fondamentale de concilier l'agriculture et l'élevage extensif dans un espace qui se ferme (environ $80 \%$ du terroir sont des agdals). Cette question est d'autant plus importante que les hihis voient maintenant en l'élevage caprin une expression de richesse.

Cette nouvelle situation, caractérisée d'une part, par un accroissement des surfaces cultivées et d'autre part, par une augmentation des effectifs des troupeaux, relève de ce que Dugue (1999) qualifie de stratégies contradictoires.

La raréfaction de plus en plus de terres cultivables utilisables pour l'agriculture ou l'élevage, conjugués avec les besoins croissants d'une population en constante augmentation ont abouti à des pressions insupportables sur l'espace hihi. Dans cette région où la population qui s'accroît sans cesse non seulement ne dispose que d'un capital restreint de terre arable mais provoque même la diminution de ce capital.

Ainsi, l'espace agricole et forestier perd sa vocation première, celle d'assurer les objectifs vitaux de chaque foyer. Les exploitations étant de moins en moins aptes à faire vivre les familles, le recours à d'autres activités devient presque obligatoire. Certains agriculteurs ayant les moyens d'acheter des céréales se démotivent et cessent de semer certaines de leurs parcelles. D'autres se découragent et partent définitivement.

Ainsi, pour trouver son équilibre économique, cette région a dû envoyer une bonne partie de sa population à l'extérieur à la recherche d'autres revenus. Ce phénomène a touché tous les aspects de la vie économique et sociale et y a apporté des transformations très importantes. Ces changements socio-économiques et spatiaux ont eu pour effet la désorganisation de l'espace hihi et une dégradation inquiétante de l'arganeraie. C'est le faisceau de tous ces indices qui laisse à penser que cet écosystème n'est plus en équilibre.

Cette évolution a favorisé une certaine prise de conscience vis-à-vis de la protection de l'arganier allant dans le sens de la théorie de conservation dite « gagnant-gagnant» (Aziz et al., 2013). C'est pourquoi, depuis une vingtaine d'années, des réflexions sont menées et des efforts sont déployés pour définir les conditions d'un développement réellement durable. Pour les développeurs, l'huile d'argane est sûrement la production sur laquelle pourrait reposer un projet de développement socio-économique (Chaussod et al., 2005). Appuyés par plusieurs organismes de développement nationaux et internationaux, différents projets ont orienté leurs stratégies vers l'approche «femmes dans le développement» dont l'objectif est de promouvoir la participation des femmes dans les processus de développement à travers la création des coopératives d'extraction de l'huile d'argane.

Soutenue par battage médiatique extrême, l'huile d'argane a vu sa demande accroître rapidement sur divers marchés, au Maroc mais surtout à l'exportation, en particulier depuis la reconnaissance scientifique et le renforcement de la dimension scientifique de l'usage de l'huile d'argane dans le domaine cardiovasculaire et en dermatologie.

L'intérêt grandissant pour ce produit de terroir, a conduit les autorités marocaines à promouvoir un processus de qualification de l'huile qui s'est concrétisé par la création en 2009 de l'IGP Argane (Indication Géographique Protégée «Argane»). Cette dernière est présentée par les pouvoirs publics comme un moyen de structurer et de stimuler la filière huile d'argan, afin de stabiliser la 
valeur ajoutée sur le territoire de l'arganeraie où elle est créée par le travail des populations locales, en particulier celui des femmes dans les coopératives de production de l'huile d'argane. Toujours selon les développeurs, l'IGP est aussi le seul moyen d'éviter la délocalisation des activités tout en défendant les produits et les savoirs locaux. On attend aussi de l'IG «Argan » qu'elle protège la biodiversité de l'écosystème arganeraie. En exhortant les familles et surtout les femmes à se regrouper dans des coopératives de production et de vente d'huile d'argane, les développeurs souhaitent qu'elles soient moins dépendantes de la forêt pour leur subsistance, réduisant ainsi sa dégradation (Dahou et al., 2011). Cependant, si cette finalité écologique était recherchée par les développeurs à travers cette valorisation économique des produits de l'argane (Simenel et al., 2009), l'évolution du marché de l'huile d'argane a eu d'autres conséquences négatives et des effets contraires visiblement non envisagés sur l'environnement, la vie socioéconomique et culturelle au niveau de l'arganeraie des Haha.

\section{L'EFFET BOOMERANG DES ACTIONS DES DÉVELOPPEURS}

Si traditionnellement, l'huile d'argane circulait dans un cercle d'intimes (Jabiot, 2007) et familial, aujourd'hui, avec la valorisation de la ressource, une dizaine de sociétés industrielles commercialisent l'essentiel de leur production hors de l'arganeraie, dont une partie importante destinée à l'export. L'ouverture du marché au niveau national et international et la création des coopératives féminines d'extraction de l'huile d'argane a eu pour effet principal une soudaine flambée du prix de l'huile et des amandons (figure 15) (Aziz et al., 2013). En effet, ces coopératives sont devenues de grands demandeurs de fruits d'argane étant donné qu'elles doivent produire plus d'huile d'argane pour répondre à une demande nationale et surtout internationale en plein essor. Cela s'est répercuté sur les prix de vente, lesquels sont décuplés par rapport à ceux des marchés locaux (Romagny et Guyon, 2009).

Le marché de l'huile d'argane cosmétique a également explosé depuis la fin des années 1990 entrâ̂nant une recrudescence d'intérêt économique pour l'huile d'argane, de la part des populations locales qui s'est traduite par l'extension spatiale des aires

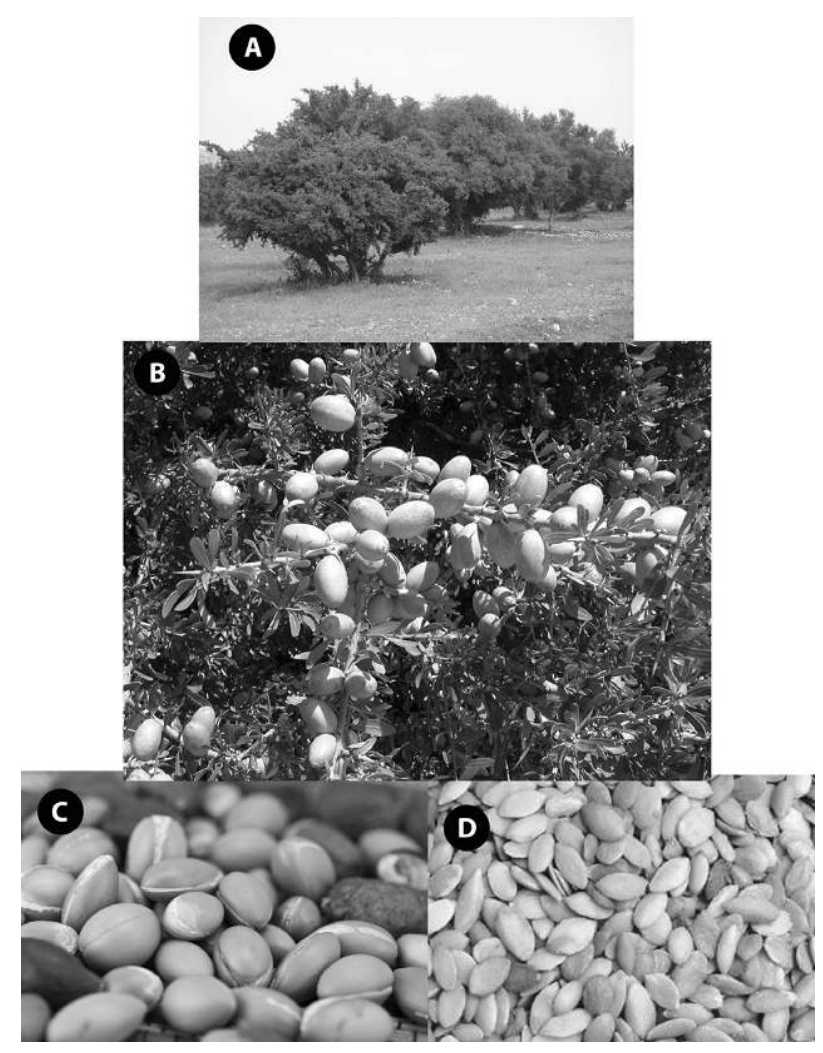

Figure 15 : L'arganier (A) donne un fruit (affiache) (B), après dépulpage de ce dernier, on obtient la noix $(a k k a)(\mathrm{C})$, cette dernière contient des amandons (tiznine) (D) qui seront écrasés pour en extraire l'huile d'argane (clichés : [A] M. G. Bernard. [B] J.-P. Peltier. [C] argan-export.com. [D] lnt.ma)

The argan tree (A) gives a fruit (affiache) (B), after removing the pulp of the latter, we obtain the nut (akka) (C) which contains the kernels (tiznine) (D) which will be crushed to extract the argan oil

de cueillette. Bien entendu, les populations locales ne peuvent pas rester indifférentes et insensibles aux gains engendrés par l'augmentation du prix des produits de l'arganier. De plus en plus de familles se mettent alors à vendre les produits de l'arganier (huile, noix et amandons), en empruntant les réseaux marchands du souk (Simenel et al., 2009). Les familles vivant dans la pauvreté chercheront à tirer profit de ce filon en ramassant tous les fruits d'argane (affiache) se trouvant dans l'arganeraie afin de les vendre aux intermédiaires. C'est ainsi que le nombre de familles collectant les fruits manuellement dans les arganeraies a plus que doublé depuis les années 1990 ainsi que la quantité de fruit collecté par famille.

En effet, pour la récolte des noix (affiache), les femmes ne se contentent plus des arganiers situés dans leurs champs ou dans leurs agdals; elles se 
mettent à cueillir les fruits situés partout dans l'arganeraie et de manière braconnière se comportant en prédatrices vis-à-vis de l'arganier mettant ainsi en péril la régénération naturelle de l'arganeraie. Initialement, il ne nous est pas apparu judicieux d'avancer un lien de causalité entre l'exploitation des arbres pour la production d'huile d'argane et la dégradation de l'arganeraie : après tout, la vie d'un arbre n'est pas conditionnée par l'utilisation de ses fruits. Mais, c'était sans penser au fait que le fruit (affiache) (figure 15) est essentiel à la reproduction naturelle de l'arganier puisqu'il initie le cycle de germination. Or, la production d'huile d'argane mobilise presque tous les affiaches disponibles, empêchant les graines de tomber et de germer. En effet, les familles vont, soit directement prélever les fruits sur les arbres, soit les ramasser sur le sol. Autrement dit, les probabilités qu'il reste des graines susceptibles de faire germer un nouvel arbre sont faibles.

Avec l'accroissement des actes de vols de fruits d'argane et l'évolution de leur valeur on assiste même à l'apparition de la pratique du gaulage qui se fait à l'aide de bâtons ou de pierres. Partout dans l'arganeraie, on voit des gens gauler les fruits, et ceci est dû au fait que les fruits gaulés se vendent plus cher, et ils sont donc plus rentables, car ils ont un poids plus élevé que ceux ramassés au sol qui sont desséchés. Cette pratique nuit gravement à la production de fruits de l'année suivante puisqu'elle provoque la chute des bourgeons floraux ou des fleurs déjà en place. Il occasionne aussi des blessures, portes d'entrée de maladies (Aziz et al., 2013), mettant ainsi en danger la régénération naturelle des arganiers.

D'un système d'apprivoisement de l'arbre et de la forêt, on passe ainsi à un système d'annihilation où la cueillette devient la seule pratique légitime (Simenel et al., 2009). Une cueillette-braconnage, conséquence indirecte de la montée des prix des produits d'argane.

Sur le plan de l'élevage, force est de constater que l'anoblissement de l'arganier comme produit de luxe mondialisé se fait aux dépens de la gent caprine, privée de sa friandise favorite (Semal, 2006). Avec les exigences de qualité des marchés intérieurs et internationaux, la majeure partie des fruits est collectée manuellement puisque les graines collectées à travers les chèvres sont de moindre qualité (Aboudrare et al., 2009). En effet, les fruits d'argane sont souvent mangés par les chèvres, qui en régurgitent les aman- dons après ingestion. Si ces amandons sont utilisés pour l'extraction de l'huile, celle-ci s'en trouve fortement altérée, aussi bien dans ses propriétés que par son odeur. En fait, la mondialisation des produits de l'argane a engendré deux dynamiques contradictoires. La première va dans le sens d'une reconfiguration de la composition du troupeau local. Soit en reconvertissant le cheptel caprin en ovin ou en délaissant définitivement l'élevage du fait de la difficulté à trouver des bergers, et de la prolifération des agdals. Cela se traduit par la multiplication des enclos, ce qui représente une période très critique pour les éleveurs, vu que leurs troupeaux ont un accès très limité aux espaces de pâturage, puisqu'ils sont exclus des agdals, ce qui a pour corollaire une assez forte concentration des troupeaux sur les mouchaâs. La deuxième dynamique est radicalement à l'opposé de la première puisque les familles qui ont bénéficié du boom d'argane, en collectant plus d'affiaches, ont davantage investi dans l'élevage caprin. Ceci est totalement contradictoire avec la théorie de conservation étant donné que le bénéfice tiré des produits de la forêt se transforme en une menace pour celle-ci à travers l'impact négatif que constituent les chèvres pour les arganiers (Aboudrare et al., 2009). Tout cela exacerbe la compétition pour l'accès aux ressources et aux espaces entre les usagers, notamment entre éleveurs et agriculteurs, surtout les titulaires du droit de récolte des fruits d'argane.

Sur le plan socioculturel, la marchandisation de l'arganier a induit des effets énormes à commencer par le changement de l'image, du symbole et de représentations vis-à-vis de l'arganier. Depuis la flambée des prix des produits de l'arganier, la relation de la population à cet arbre est devenue une relation marchande. L'argane est devenu pour nombreuses familles, une source de revenus, qui n'a pas seulement bouleversé les rapports entre l'homme et la femme en ce sens que cette dernière participe de plus en plus et activement à la prise de décisions familiales, ce qui représente un acquis social certes extraordinaire, mais il a aussi créé des tensions entre les membres de la population. En effet, comme le notent Michel Aglietta et André Orléan à propos de la marchandisation : «Derrière toute marchandise nouvelle, même la plus banale, se joue toujours une redéfinition des rapports sociaux antérieurs " (Aglietta et Orléan, 2002, p. 46). En effet, avec la marchandisation de l'arganier, les conflits ont pris 
une grande ampleur, traduisant ainsi les demandes multiples et les pressions concurrentes s'exerçant sur les ressources naturelles dans l'arganeraie.

Les habitudes alimentaires ont été, elles aussi, affectées par la spectaculaire évolution du marché de l'huile d'argane. Si cette dernière était jusque très récemment, un ingrédient principal des plats traditionnelles, elle est devenue un élément rare ou absent chez plusieurs familles. Devant la hausse de son prix, les gens préfèrent la vendre et la remplacer par l'huile industrielle (Aziz et al., 2013).

L'évolution du marché de l'huile d'argane a aussi touché le savoir-faire ancestral qui commence à s'éroder. Le procédé manuel d'extraction de l'huile d'argane illustre bien les conséquences de cette évolution sur le savoir local. En fait, nombreuses sont les femmes qui n'extraient plus l'huile d'argane chez elles, préférant vendre les amandons (tiznine) ou la noix $(a k k a)$ qui rapportent beaucoup plus d'argent que la production de l'huile. La multiplication des coopératives de production de l'huile d'argane participe, elle aussi, à l'extinction de ce savoir local : pour nombre de ces coopératives, l'extraction de l'huile se fait mécaniquement par des machines et la seule étape qui reste manuelle est le concassage.

Il est clair que le boom du marché d'argane n'a pas instauré un comportement collectif de conservation des arganeraies, mais, au contraire, s'ajoutant aux effets conjugués de l'accroissement démographique, de la sécheresse et de la pauvreté, il accentue plus la dégradation de l'arganeraie qui s'est intensifiée au cours des deux dernières décennies. Des années durant lesquelles le coût de la vie a augmenté de près de $25 \%$.

Dans un contexte marqué par la croissance démographique, la sécheresse et par un niveau de pauvreté très élevé (Faouzi, 2003), l'évolution économique de la filière de l'huile d'argane, laisse donc planer une menace sérieuse sur l'équilibre écologique des arganeraies dont la pérennité est mise en danger par une exploitation supérieure aux capacités de production (Nouaim, 2005).

\section{LE DÉVELOPPEMENT DURABLE DE L'ARganeraie des HaHa PASSE PAR L'INTERVENTION ÉTATIQUE}

La région des Haha est précisément une région où la croissance démographique et ses incertitudes pèseront lourd sur tous les plans d'aménagement au cours des prochaines décennies. La pression sur les ressources naturelles va inévitablement accélérer la dégradation de l'arganeraie qui se traduit par l'absence de régénération naturelle. Les besoins en bois pour la cuisine mais aussi pour le chauffage ne peuvent que croître, car ce combustible a le grave défaut d'être gratuit dans les campagnes. La pression sur la terre cultivée conduira à de nouveaux défrichements de l'arganeraie et à une dégradation des sols particulièrement vulnérables. Le surpâturage est tout aussi inévitable : l'effectif des troupeaux, toutes espèces confondues, augmente aussi vite et parfois plus vite que celui de la population.

La pauvreté qui s'exprime par l'incapacité d'accès aux moyens matériels pour la majorité des agriculteurs, est une conséquence de l'insuffisance des revenus agricoles. Ces micro-exploitations, qui sont souvent incapables de développer une économie d'échange, arrivent ainsi mal à se transmettre.

Les conflits sociaux qui surgissent de l'usage des terres collectives sont de plus en plus nombreux. La surexploitation entraînant l'épuisement des réserves en eaux souterraines provoque la faillite de plusieurs exploitations agricoles.

Les taux élevés du chômage dans la région et l'hémorragie migratoire qui en résulte et qui concerne particulièrement la force active, risque d'approfondir la rupture du transfert du savoir-faire traditionnel dans la région et de compromettre ainsi la durabilité de l'écosystème d'arganier.

L'intervention étatique dans cette région reste très limitée. Les efforts de l'État en matière de développement matérialisés par la création des coopératives féminines pour atténuer l'amplitude de ces contradictions restent très insuffisants. Or, dans cette région, la seule alternative de développement réside dans une intervention de l'État en faveur des fellahs dépourvus de moyens financiers, qui doit se traduire par une politique d'aide financière sous forme de crédit. Mais en réalité, le Crédit agricole, qui est en principe destiné au financement des travaux agricoles et à l'achat des animaux de trait ainsi qu'au creusement des puits, se heurte au problème de seuil de la taille de la propriété terrienne. Les petits propriétaires, et surtout les paysans sans terres, restent privés de ces aides, qui en revanche ne profitent, pratiquement, qu'aux grands exploitants. 
Mais l'action la plus importante à nos yeux, est l'amélioration du niveau de vie de la population et la lutte contre la pauvreté. L'arganeraie des Haha reste à l'écart de la croissance économique et du progrès social. Partout où nous avons enquêté, nous avons noté les faibles revenus de la population, la faiblesse de la scolarisation, le manque d'infrastructure de base, l'exacerbation de l'exode rural, etc.

L’aire géographique des Haha est une région originale et intéressante par son cadre environnemental, sa position géographique entre trois importantes zones touristiques (Essaouira, Marrakech et Agadir), par son riche passé historique et par ses paysages de carte postale. L'arganeraie des Haha, qui constitue l'arrière-pays de ces métropoles touristiques, peut répondre à d'autres types de demande touristique comme l'écotourisme, le tourisme culturel et le tourisme de montagne.

L'arganeraie des Haha est l'espace d'une civilisation importante. "Les vallées et les oasis irriguées sont des musées vivants de pratiques agraires ancestrales. La valorisation des spécialités culinaires et des produits agricoles comme produits du terroir peuvent être un atout pour ce tourisme » (El Fasskaoui, 2009, §58).

Le développement de ces nouvelles approches touristiques requiert de véritables plans d'aménagement (infrastructures routières, structures d'accueil légères et adaptées, développement de la politique des gîtes chez l'habitant). Pour maintenir la population sur place, les pouvoirs publics doivent aussi encourager et relancer l'artisanat local, créer des écoles de formation professionnelle pour assurer une meilleure formation des jeunes (El Fasskaoui, 2009).

L'amélioration du bien-être des populations passe aussi par la recherche de l'eau. Des solutions existent, au prix de quelques années de recherches et de financement adéquats, pour dessaler l'eau de mer avec des techniques moins coûteuses. La recherche de nouvelles ressources en eau constitue un domaine prioritaire, pour pallier l'érosion des sols et répondre aux besoins croissants résultant de la croissance démographique. Toutes les prévisions montrent qu'au cours des prochaines années les techniques actuelles ne permettront plus de répondre aux très graves pénuries de l'eau.

Un développement durable concerne tous les aspects de la vie d'une région, ce qui revient à dire qu'il faut mettre l'accent sur des changements politiques, économiques et sociaux. Et ce, pour développer ou, du moins, conserver ce patrimoine marocain : l'arganier.

\section{Conclusion}

Apparemment, il est difficile d'imaginer que la disparition de l'arganeraie marocaine soit un réel problème. Au premier abord, on pourrait penser qu'elle ne représente pas un enjeu crucial pour la survie des populations berbères ni une priorité dans la préservation de l'environnement. On pourrait aussi penser que la réduction des surfaces des arganeraies n'affecterait que le marché de l'huile d'argane, qui bénéficie plus aux grandes firmes exportatrices qu'aux populations locales. Pourtant l'arganeraie compte beaucoup plus qu'on ne le pense pour les populations berbères mais aussi pour tout le Maroc, en tant que patrimoine naturel unique mais aussi en raison du rôle essentiel qu'elle joue dans l'équilibre environnemental et de par sa contribution à l'économie de la région (Martin, 2012).

La disparition de l'arganier entraînerait inévitablement la disparition de plusieurs espèces, provoquant une diminution de la biodiversité dans la région laissant place à la dégradation des sols, participant ainsi à l'aridification du paysage. Cela a des conséquences sur l'ensemble de l'écosystème qui constitue un équilibre dans lequel l'arganier joue un rôle prépondérant.

Ce sont non seulement les productions liées à l'arganier qui seraient touchées par sa disparition, mais aussi l'ensemble des cultures, principalement céréalières. Sans l'arganier, la fertilité des sols sera mise en cause et il sera bien plus difficile de cultiver des terres qui laissent progressivement la place au désert. La disparition des arganiers est donc un enjeu pour l'ensemble des agriculteurs, dont les cultures sont toutes menacées par le phénomène de désertification.

La gestion traditionnelle de l'arganeraie est la première à remettre en cause pour rendre l'arganeraie soutenable. L'arganeraie a besoin que son modèle socio-économique évolue et adapte son fonctionnement traditionnel aux évolutions récentes.

Ces dernières interrogent aujourd'hui la durabilité du fonctionnement socio-économique traditionnel de l'arganeraie qui est caractérisé par des coutumes 
reproduites de générations en générations et que l'on peut qualifier d'anachroniques en relation avec des croyances et des habitudes jamais remises en cause (Martin, 2012). Car, si l'arganeraie a toujours fonctionné de façon coutumière, la question de sa non-durabilité n’avait jamais vraiment été posée jusqu’à maintenant.

L'origine anthropique de la dégradation des arganeraies n'est plus à démontrer. Liens de causalité entre le fonctionnement traditionnel de la région et le phénomène de déforestation sont évidents : l'intensification de l'activité économique de l'arganeraie s'opère dans un cadre qui, lui, n'évolue pas. Ce lien de causalité a été mis en évidence par plusieurs études (Bellefontaine et al., 2012, Martin, 2012; Bourbouze et El Aïch, 2005 ; Faouzi et Martin 2014; Nouaim, 2005). Penser que les traditions et le savoir-faire ancestral liés à l'arganier auraient permis d'adapter l'activité à l'écosystème de ce dernier est légitime. Mais le constat est tout à fait différent.

Nous avons pu constater que l'effet escompté de la valorisation économique de l'arganier était loin d'être réalisé. La forte demande pour les produits de l'arganier et la flambée des prix suite à la forte augmentation de la valeur de l'huile d'argane ont reconfiguré la vie socioculturelle locale avec l'apparition de nouveaux acteurs, l'effritement des traditions et perte du savoir-faire ancestral, le déséquilibre du partage de la richesse, etc., aboutissant ainsi à un changement de la représentation des populations vis-à-vis de l'arbre, qui n'est pas sans impact sur le système agraire (augmentation du ratio moutons/ chèvres, gaulage, etc.) (Bellefontaine et al., 2012).

L'arganeraie est beaucoup plus qu'une simple forêt naturelle, c'est une production vivante, une construction humaine. C'est donc l'arganeraie en tant que produit social qu'il faut préserver, bien plus que l'arganier lui-même (El Aïch et al., 2005). Il est donc nécessaire de promouvoir un développement réellement durable qui présuppose un souci d'équité et de justice sociale, ainsi que l'amélioration des conditions de vie de la population et la lutte contre la pauvreté.

La région renferme d'énormes potentialités (atouts naturels, écologiques et patrimoniaux, etc.) lui permettant de promouvoir les secteurs productifs orientés vers l'export (exemple les dérivés de l'arganier), et les secteurs d'activité à travers la promotion de l'écotourisme. Ceci, nous conduit à repousser cette vision fataliste et déterministe qui consisterait à penser que les données naturelles du milieu rural des Haha portent en elles les graines de la pauvreté et de la migration.

\section{Glossaire}

(a) : signifie que le mot d'origine est arabe

(b) : signifie que le mot d'origine est amazigh

Aâcbeb (a) : formation végétale herbacée des régions désertiques, constituée de plantes éphémères qui poussent après la pluie et qui servent de pâturage.

Affiache ou afiach (b) : fruit de l'arganier.

Agdal (b) : dans l'arganeraie des Haha, l'agdal désigne la période de la mise en défens de la forêt qui correspond à la période de la maturation des noix d'argane.

Akka (b) : noix d'argane qui contient les amandons (tiznine).

Amazigh/Imazighen (b) : le terme «amazigh » signifie Homme libre. C'est le terme que les populations autochtones de l'Afrique du nord se sont données depuis l'antiquité. Ce terme désigne aussi la langue sémitique des Berbères d'Afrique du Nord.

Argane (b) : nom vernaculaire désignant à la fois l'arbre, le fruit et l'huile de l'arganier.

Barkhane (a) : une barkhane est une dune de la forme d'un croissant dont les pointes sont allongées dans le sens du vent. Elle naît là où l'apport de sable est faible et sous des vents unidirectionnels.

Bled (a) : pays, région, campagne.

Caïd (a) : avant l'indépendance les tribus étaient sous le commandement des caïds; il s'agit de grands notables se trouvant au sommet de la hiérarchie tribale ou de la confédération des tribus. Ils utilisaient leurs kasbas dans leur douar comme un bureau où ils réglaient les affaires de la tribu. Actuellement le terme caïd désigne l'agent d'autorité locale nommé par le ministère de l'intérieur.

Chioukh (a) (sing. Cheikh) : amghar en berbère; le grand chef de tribu ou de la confédération tribale. Actuellement le cheikh est un fonctionnaire du ministère de l'intérieur; il est à la tête d'une ou plusieurs tribus.

Dlou (a) : aghrour en berbère; puits traditionnel à traction animale. Ce terme désigne aussi l'outre en caoutchouc ou en peau d'animaux notamment de caprins, servant à puiser de l'eau.

Douar (a) : village en Afrique du Nord. Groupement d'habitations réunissant des individus de la même lignée paternelle.

Fellah (a) : agriculteur. 
Khammès (a) : exploitant agricole lié à un propriétaire foncier par un contrat selon lequel le khemmas (métayers au $1 / 5$ e) fournit uniquement le travail contre le cinquième (khoms) de la récolte.

Melk (a) : bien en propriété privée.

Mouchâ̂ (a) : territoire du domaine forestier où, ne s'applique pas la mise en défens pendant l'agdal, où le droit de possession sur les arganiers n'appartient à personne et le droit de parcours appartient à tous les habitants du douar.

Naora (a) : puits traditionnel à traction animale.

Oued (a) : assif en berbère ; cours d'eau méditerranéen à écoulement occasionnel.

Rabaa ou r'baa (a) : métayer rémunéré au quart de la récolte.

Sanya (a) : voir naora.

Seguia (a) : canal d'irrigation.

Souk (a) : marchés ruraux et citadins qui se déroulent régulièrement une fois par semaine.

Taghate (b) : chèvre.

Tamsrieyt (b) : pièce réservée à la de réception des invités. Targant n'taghat (b) : l'arganier de la chèvre.

Tiznine (b) : amandons se trouvant à l'intérieur de la noix dure d'argane (akka). Ces amandons sont broyés pour produire l'huile d'argane.

Twiza (a) : pratique de solidarité traditionnelle dans le monde rural où une partie importante du travail agricole (labours, semailles, récoltes, moisson) faisait et fait encore l'objet de relations d'entraide.

Zaouia (a) : ce terme désigne à la fois l'établissement religieux sous l'autorité d'une confrérie musulmane, et la confrérie et toute la communauté qui se structure autour de ce centre spirituel et social.

\section{Bibliographie}

Aboudrare A., Lybbert T. J., Magnan N. 2009. Le marché de l'huile d'argan et son impact sur les ménages et la forêt dans la région d'Essaouira, Enquête diagnostic, Transfert de technologie en agriculture, Bulletin Mensuel de Liaison et d'Information du PNTTA, 175, p. 1-4.

Aglietta M., Orléan A., 2002. La monnaie entre violence et confiance, Paris, Odile Jacob, p. 253-254.

Auclair L., 1996. L'appropriation communautaire des forêts dans le Haut Atlas marocain, Cahier des Sciences Humaines, 32 (1), p. 177-194.

Aziz L., Mormont M., Allali K., 2013. Effets de la marchandisation de l'arganier sur la vie socioéconomique des populations de l'arganeraie marocaine, Vertigo - la revue électronique en sciences de l'environnement, 13 (1). DOI : 10.4000/ vertigo. 13445 .

Bahir M., Mennani A., Jalal M., Fakir Y., 2002. Impact de la sécheresse sur les potentialités hydriques de la nappe alimentant en eau potable la ville d'Essaouira (Mogador, Maroc), Science et changements planétaires/Sécheresse, 13 (1), p. 13-19.

Barathon J. J., Lechevalier C., El Abbassi H., 2005. Les oasis de la région de Tata (Maroc) : abandon de la vie oasienne traditionnelle et adaptation à la vie urbaine, Annales de géographie, 644, p. 449-461.

Bellaoui A. 1986. Le Haut-Atlas occidental quarante ans après. Revue de l'Occident musulman et de la Méditerranée, Volume 41, (41-42), 216-233.

Bellefontaine R., Pioch D., Palu S., 2012. Un nouveau départ pour la recherche relative à l'arganier, Science et changements planétaires/Sécheresse, 23 (1), p. 57-61.

Benzyane M., 1989. Estimation de la biomasse et étude de la croissance de l'arganier (Argania spinosa L. Skeels) dans le plateau de Haha (Essaouira). Mémoire de $3^{\text {e }}$ cycle, I.A.V. Hassan II, Rabat.

Bourbouze A., El Aïch A., 2005. L'élevage caprin dans l'arganeraie : l'utilisation conflictuelle d'un espace, Cahiers Agricultures, 14 (5), p. 447-453.

Chaussod R., Adlouni A., Christon R., 2005. L'arganier et l'huile d'argane au Maroc : vers la mutation d'un système agroforestier traditionnel?, Cahiers Agricultures, 14 (4), p. 351-356.

Dahou T., Elloumi M., Molle F., Gassab M., Romagny B., 2011. Pouvoirs, sociétés et nature au sud de la Méditerranée, Paris, Karthala.

Direction Provinciale de L'Agriculture d'Essaouira, 2003. Rapport technique. Confédération des Haha.

Direction Provinciale de L'Agriculture d’Essaouira, 2001. Questionnaire monographique. Commune rurale de Smimou.

Dugue P., 1999. Utilisation de la biomasse végétale et de la fumure animale: impacts sur l'évolution de la fertilité des terres en zone des savanes. Étude de cas au Nord-Cameroun et essai de généralisation. Rapport final de l'ATP : Flux de biomasse et gestion de la fertilité à l'échelle du terroir, document CIRAD-TERA 57/99.

El Aich A., Bourbouze A., Moran-Fenr P., 2007. La filière du chevreau de l'arganeraie, un produit typique et durable, Annales de la recherche forestière au Maroc, 38, p. 124-137.

El Fasskaoui B., 2009. Fonctions, défis et enjeux de la gestion et du développement durables dans la Réserve de Biosphère de l'Arganeraie (Maroc), Études caribéennes, 12. DOI : 10.4000/etudescaribeennes.3711.

Emberger L., 1955. Une classification biogéographique des climats. Recueil des travaux du laboratoire de Botanique, Géologie et zoologie, 7, 3-43, université de Montpellier.

Faouzi H., Martin J., 2014. Soutenabilité de l'arganeraie marocaine, Confins, 20., DOI : 10.4000/confins. 8842.

FAouzi H., 2013. L'exploitation du bois-énergie dans les arganeraies : entre soutenabilité et dégradation (région des Haha, Haut-Atlas Occidental, Maroc), Les Cahiers d'Outre-Mer, 262, 155-182.

FAouzi H., 2011. L'agdal dans la dynamique des systèmes agraires des arganeraies des Haha (Haut-Atlas Occidental, Maroc), Études caribéennes, 20. DOI : 10.4000/etudescaribeennes.5569. 
FAouzI H., 2012. Impact des coopératives féminines sur la préservation et la valorisation de l'arganeraie : cas de la coopérative Tafyoucht (confédération des Ait Baamrane, AntiAtlas, Maroc), Confins, 14. DOI : 10.4000/confins.7521

Faouzi H., 2007. Arganeraies, démographie, émigration et mutations, Le matin du 24 janvier, p. 6.

Faouzi H., 2003. L'arganeraie des haha : étude d'un système agraire en mutation (Haut-Atlas occidental, Maroc). Thèse de doctorat en géographie, Université Nancy 2, Nancy.

FAY G., 1987. Désagrégation des collectivités et dégradation des milieux dans le Haut-Atlas marocain, Revue de l'Occident Musulman et de la Méditerranée, n 41-42.

Féri A., 1993. Contribution à l'étude hydrogéologique et hydrogéochimique de la zone synclinale d'Essaouira (bassin synclinal d'Essaouira), Thèse de doctorat, Université Cadi Ayyad, Marrakech.

Haut Commissariat au Plan, 2004. Recensement général de la population et de l'habitat de 2004, Rabat, HCP.

Јавіот I., 2007. Entre innovation et conformité. La coopérative Tamounte d'extraction d'huile d'argan, Mémoire de master d'ethnologie, Université Paris 10 Nanterre.

Jouve P., 2006. Transition agraire : la croissance démographique, une opportunité ou une contrainte?, Afrique contemporaine, 1 (217), p. 43-54. DOI 10.3917/afco.217.54.

LÉOn L'Africain J., 1956. Description de l'Afrique septentrionale (Epaulard, Trad.), Paris, Maisonneuve, (Euvre originale publiée en 1556).

Malthus T. R., 1798. An essay on the principle of population, as it affects the future improvement of society. With remarks on the speculations of Mr. Godwin, M. Condorcet, and others writers, Londres: J. Johnson.

Martin J., 2012. La soutenabilité du développement de l'arganeraie au Maroc. Mémoire de master en Économie du déve- loppement durable, Institut d'Études Politiques/Université lumière Lyon 2.

Naggar M., Mhirit O., 2006. L'arganeraie : un parcours typique des zones arides et semi-arides marocaines, Science et changements planétaires/Sécheresse, 17 (1-2), p. 314-317.

Nouaim R., 2005. L'arganier au Maroc, entre mythes et réalités, Paris, L'Harmattan.

Person S., 1998. Targant n'Tarat ou l'arganier de la chèvre, Rapport de stage ESAT-1. CNEARC-IAMM, Montpellier.

Ponteves E. (de), 1989. L'arganier, la chèvre, l'orge : approche du système agraire de l'arganeraie dans la commune rurale de Smimou, Province d'Essaouira, Maroc, Mémoire de Fin d'Études. Diplôme d'ingénieur agronome, CNEARC, Montpellier.

Robine L., 1956. Revenus et niveaux de vie du Grand Atlas occidental, Rabat, imprimerie Thouret.

Romagny B., Guyon M., 2009. Des souks aux marchés internationaux. La valorisation économique de l'huile d'argan marocaine : un cas d'école des contradictions du développement durable, In Localiser les produits : actes du colloque international organisé par le CIRAD, l'IRD, le Mab/UNESCO et le MNHN, Paris, UNESCO.

Semal J., 2006. Vous avez dit « développement durable »?, Cahiers Agricultures, 15 (1), p. 159.

Simenel R., Michon G., Auclair L., Thomas Y., Romagny B., Guyon M., 2009. L'argan : l'huile qui cache la forêt domestique. De la valorisation du produit à la naturalisation de l'écosystème, Autrepart, 50 (2), 51-73. DOI : 10.3917/ autr.050.0051.

SulzleE C., 1962. Les dunes d'Essaouira, Revue forestière française, 15 (5), p. 401-418. 